\title{
The 2-Look TOPS Mode: Design and Demonstration with TerraSAR-X
}

\author{
Nestor Yague-Martinez, Pau Prats-Iraola, Senior Member, IEEE, Steffen Wollstadt \\ and Alberto Moreira, Fellow, IEEE
}

\begin{abstract}
Burst-mode acquisition schemes achieve wide coverage at the expense of a degraded azimuth resolution, reducing therefore the performance on the retrieval of ground displacements in the azimuth direction, when interferometric acquisitions are combined. Moreover the azimuth varying line-of-sight can induce discontinuities in the interferometric phase when local azimuth displacements are present, e.g., due to ground deformation. In this contribution we propose the interferometric 2-look TOPS mode, a sustaining innovation, which records bursts of radar echoes of two separated slices of the Doppler spectrum. The spectral separation allows to exploit spectral diversity techniques, achieving sensitivities to azimuth displacements better than with StripMap, and eliminating discontinuities in the interferometric phase. Moreover some limitations of the TOPS mode to compensate ionospheric perturbations, in terms of data gaps or restricted sensitivity to azimuth shifts, are overcome. The design of 2-look TOPS acquisitions will be provided, taking the TerraSAR-X system as reference to derive achievable performances. The methodology for the retrieval of the azimuth displacement is exposed for the case of using pairs of images, as well as for the calculation of mean azimuth velocities when working with stacks. We include results with experimental TerraSAR-X acquisitions demonstrating its applicability for both scenarios.
\end{abstract}

Index Terms-2-look TOPS, burst-mode, wide coverage, Synthetic Aperture Radar (SAR), SAR Interferometry, TerraSAR-X.

\section{INTRODUCTION}

Current remote sensing satellites are operated following near-polar orbits. Since radar systems measure distances in the line-of-sight direction, they are very sensitive to ground displacements in the East-West (E-W) and vertical directions. By combining different geometries, a 3-D deformation field can be obtained [1], however the sensitivity to displacements in the North-South (N-S) direction remains low. A common procedure to enhance the sensitivity in along-track direction, and therefore to the N-S direction, is to apply correlation techniques exploiting the imaging capability of SAR. This has been done extensively with images acquired in StripMap mode for different applications, as e.g., tectonics [2] or the determination of glaciers ice flow [3].

With the high demand for wide-area coverage data from the scientific community, new SAR acquisition modes have been implemented on current systems or are under development. The ScanSAR mode [4] was the first to offer wide coverage by recording subsets of radar echoes over multiple

The authors are with the German Aerospace Center (DLR), Oberpfaffenhofen, D-82234, Germany. (e-mail: nestor.yague@dlr.de) subswaths. ScanSAR presents some limitations related to azimuth-dependent ambiguities and SNR, and the so-called scalloping effect, that have been overcome by the Terrain Observation by Progressive Scans (TOPS) mode [5]. However, both of them present a reduced azimuth resolution, due to the burst-mode acquisition nature, impairing the accuracy of correlation techniques in this direction, since the sensitivity depends on the resolution cell size [6]. In order to solve this paradox two strategies are possible: the first one consists on achieving a wide swath keeping high azimuth resolution. This can be fulfilled by employing multichannel systems like the proposed High Resolution Wide Swath (HRWS) SAR mode [7]. In the last years an important number of concepts and techniques have been developed following this philosophy. Several works have been published which deal with the use of digital beamforming techniques [8], the use of multiple azimuth channels [9] [10] and the staggered SAR concept [11], which employs non-constant acquisition pulse repetition intervals to avoid blind ranges when digital beamforming techniques are applied in elevation.

A second option, which is the focus of this paper, consists in employing conventional wide swath burst-modes acquiring two looks. The achieved coverage is the same as the one provided by single look modes at the expense of a degradation of the azimuth resolution by a factor of two, maintaining however the number of looks for a given product resolution. The benefit of this strategy lies on the possibility to exploit spectral diversity techniques [12][6], improving significantly the sensitivity to azimuth surface displacements. This approach can be also understood from a geometrical point of view in that two lines of sight are almost simultaneously obtained [13] using a single platform. The concept of this innovative acquisition mode, which exploits a single phased array antenna to record two separated slices of the spectrum, was demonstrated with TerraSAR-X in 2015 employing ScanSAR [14] and in 2016 with TOPS [15]. The focus of this contribution is set on the 2-look TOPS mode which, besides the known benefits over ScanSAR, allow tuning the sensitivity to the azimuth displacements. In opposition to HRWS techniques, the 2-look mode keep a low resolution in the azimuth direction, which does not suppose a limitation for geophysical applications, where high spatial resolution is not required.

The paper is structured as follows. Section II introduces the 2-look TOPS mode, illustrating its spectral properties and providing the timeline equations. Two designs are presented, the first, TOPS2, based on the desired azimuth resolution and 


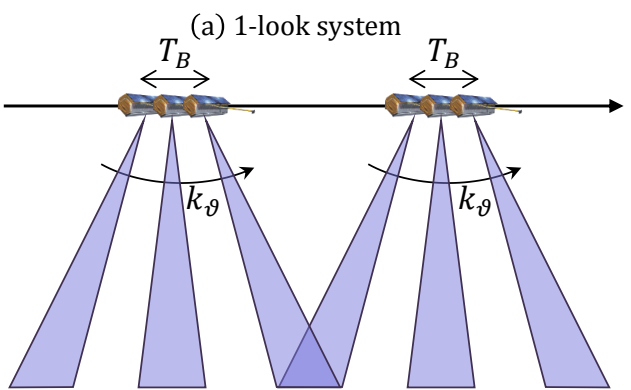

(b) 2-look system

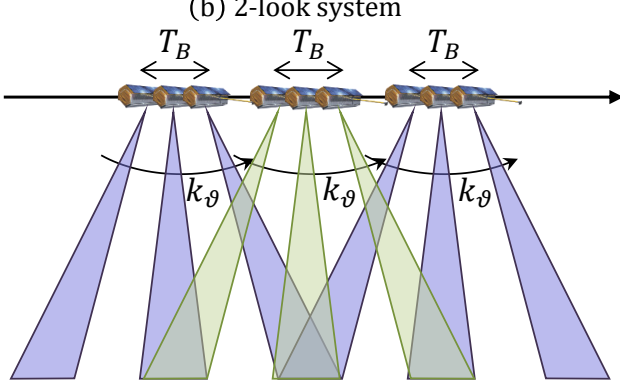

Fig. 1. (a) Schematic operation of a TOPS system (1 look) and a (b) 2-look TOPS system. The antenna is steered in azimuth from backward to forward at a rate $k_{\vartheta}$. In a 1-look system each target is observed only once (except eventually at the burst overlapping areas), a 2-look system allows illuminating each target with two different squint angles.

the second, TOPS2+, based on a design driven by the azimuth sensitivity. In section III, the evaluation of the performance of the mode is provided, including the effects of the antenna sweeping, ambiguities, residual scalloping and interferometric performance. Section IV focuses on the sensitivity that can be achieved for pairs of images and for time-series, considering the effect of the troposphere. Some comments related to the ionospheric phase screen are, as well, provided. In section $\mathrm{V}$ the methodology for the generation of 2-look interferograms, the retrieval of azimuth local displacement for pairs and the retrieval of the mean azimuth velocity for time-series is exposed. Section VI provides experimental results with TerraSAR-X: in the first place, an interferometric pair of acquisitions over the Petermann glacier, Greenland, shows the high potential of the 2-look mode to eliminate phase discontinuities of the InSAR phase for fast-moving sites. The second scenario corresponds to a slow-deforming area over Balochistan, Pakistan. The evaluation of approximately two years of acquisitions demonstrates the capability of the mode to retrieve a post-seismic signal with accuracies for the mean azimuth velocity of just a few $\mathrm{mm} / \mathrm{month}$.

\section{THE 2-LOOK TOPS MODE}

The TOPS mode has been designed to illuminate each target by the entire azimuth antenna pattern performing an azimuth scanning. The acquisition takes place by recording bursts of echoes, which correspond to a portion of the synthetic aperture. The beam is switched cyclically in elevation in order to map multiple subswaths and to obtain wide coverage.
TOPS overcomes this way the space-varying properties of the azimuth antenna pattern in ScanSAR, which leads to a periodical modulation of the amplitude of the SAR signal (scalloping effect), i.e., the resolution, ambiguity ratio and Noise Equivalent Sigma Zero (NESZ) [5]. In section II-A the fundamental reasons for choosing a 2-look mode are provided. In sections II-B and II-C the design equations of such approach are provided.

\section{A. Rationale}

In order to be able to retrieve a two-dimensional ground deformation field, at least two lines of sight are needed, as proposed in the dual-beam interferometer [16]. This concept achieves sensitivity in the across- and along-track direction by employing two antennas, one mechanically oriented with a certain squint forward and a second one oriented backward. The squint angles of the resulting lines of sight are chosen usually symmetric around the zero-Doppler plane. When moving to a classical spaceborne system in which a single phased array antenna is available, the rotation of the antenna of the TOPS mode can be used, not only to illuminate each target with the whole antenna pattern, but to also record each target observed by two separated Doppler frequencies, which corresponds to observe each target on ground with two (varying) lines of sight.

The combination of the two previous ideas with the TOPS operation mode can be fulfilled by designing the scanning timeline for a 2-look system, that achieves two quasi-simultaneous lines of sight with spatial diversity. In this subsection we restrict the 2-look concept explanation to one subswath, the extension for several subswaths is immediate. Fig. 1 illustrates the operation mode of a 2-look TOPS system compared to a 1-look TOPS system, henceforth referred as TOPS. On the top of Fig. 1 the acquisition of two bursts for a TOPS system is depicted. Employing one look, each target on ground is observed with just one line of sight. The bottom part of the figure corresponds to a 2-look system, in which the scanning timeline allows to illuminate each target with two different lines of sight.

Unlike the dual-beam interferometer, the 2-look TOPS mode obtains two time-varying lines of sight, however the spatial diversity between both remains constant over the acquisition. This can be better understood by comparing the time-frequency diagrams for TOPS and 2-look TOPS depicted in Fig. 2. The top part of the figure corresponds to the conventional 1-look system, which illustrates the acquisition of two consecutive bursts of the same subswath for a certain range, presenting each target a Doppler rate, $k_{\mathrm{a}}$. The antenna is rotated in the azimuth direction originating a linear frequency variation of the burst, of duration $T_{\mathrm{B}}$, at a rate $k_{\text {rot }}$. The SAR processing at zero-Doppler geometry causes that the focused burst, of duration $T_{\text {focused }}$, exhibits a linear frequency variation with a rate $k_{\mathrm{t}}$. The cycle time or interburst time, $T_{\mathrm{R}}$, is the elapsed time between bursts of the same subswath. Two targets, $P_{1}$ and $P_{2}$, are displayed at their zero-Doppler 

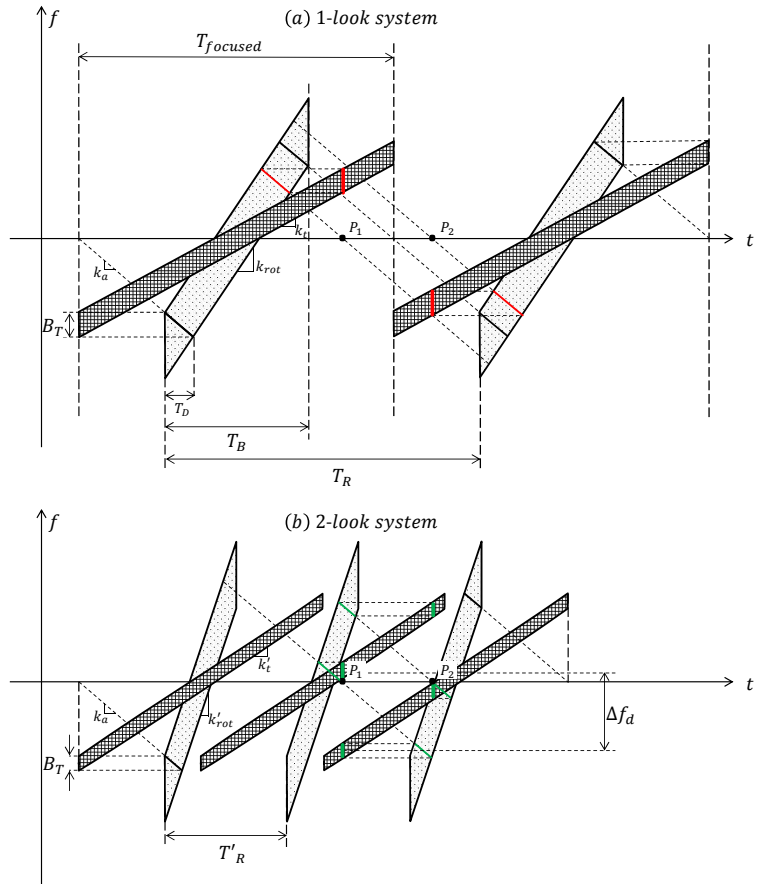

Fig. 2. (a) Time-frequency diagram of a TOPS system, which shows two bursts of the same subswath at a certain range. Each target presents a Doppler rate, $k_{\mathrm{a}}$. The antenna is rotated in the azimuth direction originating a linear frequency variation of the burst, of duration $T_{\mathrm{B}}$, at a rate $k_{\mathrm{rot}}$. The SAR processing at zero-Doppler geometry causes that the focused burst, of duration $T_{\text {focused, }}$, presents also a linear frequency variation, with rate $k_{\mathrm{t}}$. The dwell time, $T_{\mathrm{D}}$, is the time interval in which a target on ground is illuminated with the main lobe, and results on a bandwidth $B_{\mathrm{T}}$. The cycle time or interburst time, $T_{\mathrm{R}}$, is the elapsed time between bursts of the same subswath. Two targets, $P_{1}$ and $P_{2}$, are displayed at their zero-Doppler position indicating the portion of the (once) covered raw and focused data Doppler spectra. (b) Time-frequency diagram of a 2-look TOPS system. In this case the cycle time, $T_{\mathrm{R}}^{\prime}$, allows to map each target with two portions of the spectrum as can be seen for targets.

position indicating the portion of the (once) covered raw and focused data Doppler spectra. The bottom part of the figure refers to a 2-look system, where three bursts are acquired. The cycle time, $T_{\mathrm{R}}^{\prime}$, allows to map each target with two portions of the spectra, as can be seen for both depicted targets, $P_{1}$ and $P_{2}$. Note that a 2-look system records two separated slices of the Doppler spectrum, whose central frequency is azimuth-dependent, however the spectral separation between looks, $\Delta f_{\mathrm{d}}$, is constant over azimuth.

According to the signal processing rationale, the spectral separation for a target on ground between two consecutive bursts (looks) can be exploited to retrieve an accurate estimation of the azimuthal motion. From an interferometric pair, two 1-look interferograms can be generated, which when combined, result in a differential interferogram whose phase is given by [12]:

$$
\phi_{\mathrm{ESD}}(r, x)=2 \pi \cdot \Delta f_{\mathrm{d}}(r) \cdot \Delta t(r, x) \text {, }
$$

where $\Delta f_{\mathrm{d}}(r)$ is the spectral separation between looks for a given range, $r$. Note that the spectral separation is independent of the target's azimuth position. $\Delta t(r, x)$ is the local misregistration (in temporal units) intended to be measured.
The subscript ESD stands for Enhanced Spectral Diversity and the spectral separation, $\Delta f_{\mathrm{d}}(r)$, can be calculated according to [17]:

$$
\Delta f_{\mathrm{d}}(r)=\left|\frac{k_{\mathrm{rot}} \cdot k_{\mathrm{a}}(r)}{k_{\mathrm{rot}}-k_{\mathrm{a}}(r)}\right| T_{\mathrm{R}}
$$

being $k_{\mathrm{a}}(r)$ the Doppler rate of a target, located at range $r$, and $k_{\text {rot }} \approx \frac{2 \cdot v_{\mathrm{s}}}{\lambda} k_{\vartheta}$ the antenna Doppler rate, where $v_{\mathrm{s}}$ is the platform velocity, $\lambda$ the wavelength and $k_{\vartheta}$ the antenna rotation rate. Since $\Delta f_{\mathrm{d}}(r)$ is significantly larger than the spectral separation achievable by taking sublooks of the available bandwidth of a 1-look system (by a factor between 5 and 12 as will be shown in section IV), the exploitation of both looks allows the retrieval of $\Delta t(r, x)$ with a higher accuracy. We will call, in general, 2-look TOPS to a TOPS system that maps each target on ground with two separated Doppler frequencies. Henceforth we employ the nomenclature TOPS2 to a 2-look system with a timeline design driven by the azimuth resolution. The timeline equations of the TOPS 2 mode are provided in section II-B. We also propose an optimization of the scanning timeline in terms of achieving a higher sensitivity to the azimuthal motion; we refer then to a 2-look TOPS system with such a scanning timeline which selects the spectral separation between looks (usually larger than the one achievable by establishing the resolution) as TOPS2+. The design equations of TOPS2+ are presented in section II-C.

A second issue that can be addressed with the 2-look acquisition concept is related to the burst-mode acquisition nature and its application for the mapping of non-stationary scenarios. The azimuth-varying line of sight during the acquisition experiments an abrupt change at the interface between bursts, which originate phase discontinuities in the presence of azimuth misregistration due to, e.g., ground motion. These discontinuities are not to be considered as artifacts but as the result of a different projection of the (same) ground displacement onto the radar lines of sight of each look [18]. However, this effect can induce some problems for the later interferometric processing steps, e.g., in case a spatial phase unwrapping is to be performed, thus it is interesting to perform an accurate local azimuth coregistration exploiting both looks. More details will be given in sections IV-B and V-A.

\section{B. Scanning timeline equations: TOPS2}

In the first place, it is convenient to recall the dependency of the TOPS image azimuth resolution with the antenna rotation rate, $k_{\vartheta}$. The azimuth pattern observed by a point target can be approximated by a sinc function and is given by [5]:

$$
G_{\mathrm{T}}(\vartheta(t)) \approx G_{0} \operatorname{sinc}^{2}\left(\frac{L v_{\mathrm{g}} t}{\lambda R_{0}} \cdot\left(1+\frac{R_{0} k_{\vartheta}}{v_{\mathrm{g}}}\right)\right),
$$

where $G_{0}$ is a constant, $\vartheta(t)$ is the antenna rotation angle as a function of the time, $t, L$ is the physical azimuth antenna 
length, $v_{\mathrm{g}}$ the beam ground velocity and $R_{0}$ the range of closest approach.

In the following we extend our analyses to multi-subswath systems indicating with the superscript $(n)$ the $n^{t h}$ subswath. The resulting azimuth resolution, $\rho_{\mathrm{az}}$, is reduced with respect to the StripMap resolution by a factor:

$$
\alpha^{(n)}=1+\frac{R_{0}^{(n)} k_{\vartheta}^{(n)}}{v_{\mathrm{g}}^{(n)}},
$$

which leads to the azimuth resolution of the TOPS mode:

$$
\rho_{\mathrm{az}}=\rho_{\mathrm{az}}^{\mathrm{SM}} \cdot \alpha^{(n)}=\frac{\lambda}{2 \vartheta_{0}^{(n)}} \cdot \frac{\left(v_{\mathrm{g}}^{(n)}\right)^{2}}{\left(v_{\mathrm{eff}}^{(n)}\right)^{2}} \cdot\left(1+\frac{R_{0}^{(n)} k_{\vartheta}^{(n)}}{v_{\mathrm{g}}^{(n)}}\right),
$$

where $\vartheta_{0}^{(n)}$ is the antenna azimuth bandwidth at $-3 \mathrm{~dB}$ and $v_{\text {eff }}^{(n)}$ refers to the effective velocity. The superscript SM indicates StripMap mode.

Following the classical approach for the design of a SAR mode driven by the desired resolution, $\rho_{\mathrm{az}}$, the steering parameters for each subswath, $n$, can be obtained using:

$$
k_{\vartheta}^{(n)}=\left(\frac{2 \rho_{\mathrm{az}} \vartheta_{0}^{(n)}}{\lambda} \frac{\left(v_{\mathrm{eff}}^{(n)}\right)^{2}}{\left(v_{\mathrm{g}}^{(n)}\right)^{2}}-1\right) \frac{v_{\mathrm{g}}^{(n)}}{R_{0}^{(n)}} .
$$

Once the steering rate has been calculated, the global 2-look TOPS scanning timeline can be obtained setting a cycle time, $T_{\mathrm{R}}$, that allows that the total bandwidth spanned in the burst duration is at least two times larger than the one spanned by a target in the interburst interval. This leads to the equations provided in [5] including a factor 2 multiplying the cycle time:

$$
\left(k_{\vartheta}^{(n)} T_{\mathrm{B}}^{(n)}-\vartheta_{0}^{(n)}\right) R_{0}^{(n)}+v_{\mathrm{g}}^{(n)} T_{\mathrm{B}}^{(n)} \geq 2 \cdot v_{\mathrm{g}}^{(n)} T_{\mathrm{R}} .
$$

As stated in [5] there is a bound on the azimuth resolution, $\rho_{\mathrm{az}}$ :

$$
\rho_{\mathrm{az}}>2 \cdot \sum_{i} \rho_{\mathrm{az}}^{\mathrm{SM}}(i)
$$

being $\rho_{\mathrm{az}}^{\mathrm{SM}(n)}$ the resolution of an equivalent StripMap acquisition for each subswath, $n$. The factor 2 is again due to the mapping of two looks.

We can see that a 2-look system has a degraded resolution by a factor of two with respect to its equivalent 1-look system. This seems to be a contradiction considering that the looks could be combined at the SAR processing stage, following a full-aperture processing approach [19] for multi-look systems. The impulse response function provided by the latter approach is, however, strongly degraded, due to the interference of both looks. Moreover, since our interest, when designing a 2-look system, is to combine the looks at the interferometric processing stage, in order to apply spectral diversity techniques, the strategy is to focus each burst (look) separately.
A second consequence of degrading the resolution by a factor of two is that, according to (6), the steering rate is increased by a factor slightly larger than two. Provided that (8) is fulfilled, choosing a finer resolution results in a lower steering rate. This implies that the bursts become longer, spanning the antenna beam larger maximum steering angles. This fact can be exploited to maximize the spectral separation, as discussed in the next section.

C. Alternative scanning timeline equations for azimuth sensitivity enhancement: TOPS2+

The original TOPS mode scanning timeline is obtained by setting the desired azimuth resolution, as usually done. We can, however, modify the design criterion in order to increase the azimuth sensitivity by maximizing the spectral separation, $\Delta f_{\mathrm{d}}$, between looks. The latter is achieved by increasing the cycle time, $T_{\mathrm{R}}$, according to (2). Longer cycle times can be obtained either by enhancing the resolution or by imposing a larger maximum steering angle, equivalently. The design of the timeline based on the maximum steering angle is appropriate for systems employing phased-array antennas, since it allows to control the maximum level of the grating lobe and therefore the level of the azimuth ambiguities.

Once the desired maximum steering angle, $\beta_{\max }$, has been set, we can write the following relation:

$$
k_{\vartheta}^{(n)}=\frac{\Delta \beta}{T_{\mathrm{B}}^{(n)}}
$$

where $\Delta \beta=2 \cdot \beta_{\max }$. Substituting (9) in (7) we obtain the TOPS2+ set of equations:

$$
\left(\Delta \beta-\vartheta_{0}^{(n)}\right) R_{0}^{(n)}+v_{\mathrm{g}}^{(n)} T_{B}^{(n)} \geq 2 \cdot v_{\mathrm{g}}^{(n)} T_{\mathrm{R}}
$$

By solving this system of equations, the burst durations, $T_{\mathrm{B}}^{(n)}$, are obtained, which establish the steering rates for each subswath, $k_{\vartheta}^{(n)}$ according to (9). The resulting azimuth resolution is given by:

$$
\rho_{\mathrm{az}}^{(n)}=\frac{v_{\mathrm{g}}^{(n)}}{B_{\mathrm{T}}^{(n)}}
$$

being $B_{\mathrm{T}}^{(n)}=k_{\mathrm{a}}^{(n)} \cdot T_{\mathrm{D}}^{(n)}$ the target bandwidth for the $(n)^{t h}$ subswath. $T_{\mathrm{D}}^{(n)}$ is its corresponding dwell time, given by:

$$
T_{\mathrm{D}}^{(n)} \approx \frac{R_{0}^{(n)} \cdot \vartheta_{0}^{(n)}}{\alpha^{(n)} \cdot v_{\mathrm{g}}^{(n)}}
$$

The maximum steering angle cannot be set arbitrarily, since the azimuth ambiguity level increases with it. Next section provides performance analyses with the two design approaches, considering the effects of sweeping the antenna beam to large steering angles. 
TABLE I

SCANNING TIMELINE PARAMETERS FOR THE TOPS, TOPS2 AND TOPS2+ MODES, USING TWO SUBSWATHS. THE MID-RANGE POSITION OF THE SUBSWATHS HAVE BEEN ASSUMED. THE VALUES IN BOLD INDICATE THE DESIGN CRITERIA TO DETERMINE THE ACQUISITION TIMELINE PARAMETERS. AN ADDITIONAL OVERLAP BETWEEN ODD/EVEN BURSTS OF $10 \%$ HAS BEEN CONSIDERED FOR THE DESIGN. THE ACHIEVABLE VALUES FOR STRIPMAP ARE INCLUDED FOR REFERENCE. THE

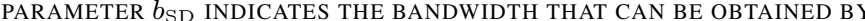
SPLITTING THE DOPPLER SPECTRUM INTO TWO SUB-LOOKS FOR

STRIPMAP AND TOPS IN ORDER TO APPLY THE SPECTRAL DIVERSITY TECHNIQUE TO MEASURE THE AZIMUTH SHIFT. FOR TOPS2 AND

TOPS2+ THERE IS NO NEED TO SPLIT THE SPECTRUM. THE LAST ROW INDICATES THE AMBIGUITY BAND OF THE AZIMUTH SHIFT. THE TERRASAR-X SYSTEM PARAMETERS HAVE BEEN USED

\begin{tabular}{lcccc}
\hline & SM & TOPS & TOPS2 & TOPS2+ \\
\hline \hline$\rho_{\text {az }}[\mathrm{m}]$ & 3.3 & $\mathbf{8 . 3}$ & $\mathbf{1 6 . 6}$ & $13.38 / 15.11$ \\
$\beta_{\max }[\mathrm{deg}]$ & - & $0.501 / 0.487$ & $0.547 / 0.532$ & $\mathbf{1 . 1}$ \\
$T_{\mathrm{B}}[\mathrm{s}]$ & - & $0.834 / 0.840$ & $0.355 / 0.358$ & $0.936 / 0.832$ \\
$T_{\mathrm{R}}[\mathrm{s}]$ & - & 1.675 & 0.71 & 1.77 \\
$k_{\vartheta}[\mathrm{rad} / \mathrm{s}]$ & - & $0.021 / 0.020$ & $0.054 / 0.052$ & $0.041 / 0.046$ \\
$\Delta f_{\mathrm{d}}[\mathrm{Hz}]$ & 1843 & 664 & $3420 / 3297$ & $8028 / 7994$ \\
$B_{\mathrm{T}}[\mathrm{Hz}]$ & 2765 & 996 & 498 & $618 / 547$ \\
$b_{\mathrm{SD}}[\mathrm{Hz}]$ & 921.67 & 332 & - & - \\
Amb. band $[\mathrm{m}]$ & $\approx \pm 2$ & $\approx \pm 5$ & $\approx \pm 1$ & $\approx \pm 0.5$ \\
\hline
\end{tabular}

\section{MODE PERFORMANCE}

This section provides a quantitative analysis of the performance of the 2-look TOPS modes regarding the level of azimuth and range ambiguities, residual scalloping effect, effects of sweeping the antenna to large steering angles and achievable interferometric performance when combining both looks.

The TerraSAR-X system has been taken as reference, employing two wide beams [20], which achieve a coverage of approximately $100 \mathrm{~km}$. Establishing a SLC azimuth resolution equal to $16.6 \mathrm{~m}$ for TOPS2 and applying Equations (6) and (7) we obtain the scanning timeline parameters detailed in Table I, where a maximum steering angle, $\beta_{\max }$, of approximately $0.55^{\circ}$ is obtained. We establish, as a matter of example, our maximum steering angle for TOPS2+ to approximately twice that of the TOPS 2 mode, i.e., $1.1^{\circ}$. The TOPS2+ timeline parameters are obtained by applying (9) and (10). The parameters for TOPS, assuming $8.3 \mathrm{~m}$ resolution, and StripMap are also included for reference. For the latter modes, the spectral diversity technique, i.e., the split of the Doppler bandwidth into two sub-looks can be applied in order to obtain the azimuth shifts. The optimal sub-look bandwidths correspond to $b_{\mathrm{SD}}=B_{\mathrm{T}} / 3$ [6], which deliver a spectral separation of $\Delta f_{d}=2 / 3 B_{\mathrm{T}}$. The corresponding values are included in the table for convenience purposes, since an intermode comparison will be provided in the sensitivity analysis in section IV.

By comparing the 1-look to the 2-look system parameters from the table we can extract following conclusions:

(i) The antenna steering rate, $k_{\vartheta}$, for TOPS2 is a factor slightly larger than two with respect to TOPS due to the $(2 \times)$ coarser resolution.

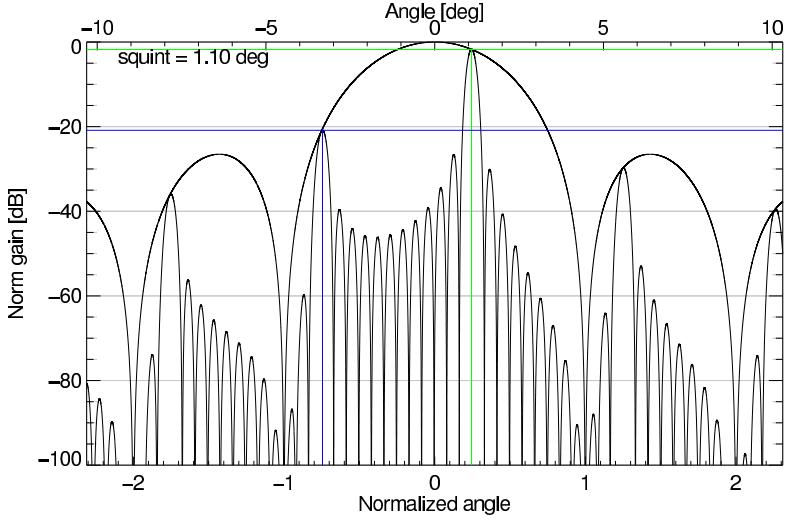

Fig. 3. Element and directivity pattern for a steering angle of 0.246 (normalized to the first null position of the element pattern), equivalent to $1.1^{\circ}$ using the TerraSAR-X antenna panel

(ii) The fact of steering to larger maximum squint angles (TOPS2+) does not have a negative impact on the SLC resolution as long as the condition imposed by (8) is fulfilled. The antenna steering rate becomes smaller than for TOPS2, due to the fact that the bursts become longer when steering the antenna beam to larger maximum squint angles.

(iii) The unambiguous range of the azimuth shifts that can be retrieved is given by $\pm v_{\mathrm{g}} /\left(2 \Delta f_{\mathrm{d}}\right)$, in meter units. Due to the higher sensitivity of TOPS2+ the ambiguity band is smaller, as indicated in the last row of Table I, so that $\phi_{\mathrm{ESD}}$ might be wrapped if the motion gradient is large.

\section{A. Effects of sweeping the antenna beam to large steering angles}

When working with phased array antennas, the maximum steering angle cannot be set arbitrarily large since the level of the azimuth ambiguities increase [21] [22]. The largest contribution to the azimuth ambiguities level is due to the so-called grating lobes, which are the periodic repetitions of the main lobe. The sinc modulation effect of the antenna element pattern tapers the level of the grating lobes, making significant only the first one.

In order to illustrate the relationship between main and grating lobe when introducing a steering angle, Fig. 3 shows the directivity and element pattern for a squint angle of $1.1^{\circ}$, equivalent to a normalized (to the first null position of the element pattern) angle equal to 0.246 . The plot has been generated by means of a numerical simulation employing the sinus cardinalis function according to the TerraSAR- $X$ antenna dimensions, number of elements and wavelength, detailed in Table II. We can see that for this squint the level of the grating lobe is higher than the first side lobe. The limiting parameter for setting the maximum steering angle will be given by the requirement value for the Ambiguity-to-Signal Ratio (ASR). Sections III-B and III-C provide a discussion on the maximum steering angle and optimal Pulse Repetition Frequency (PRF) selection. 


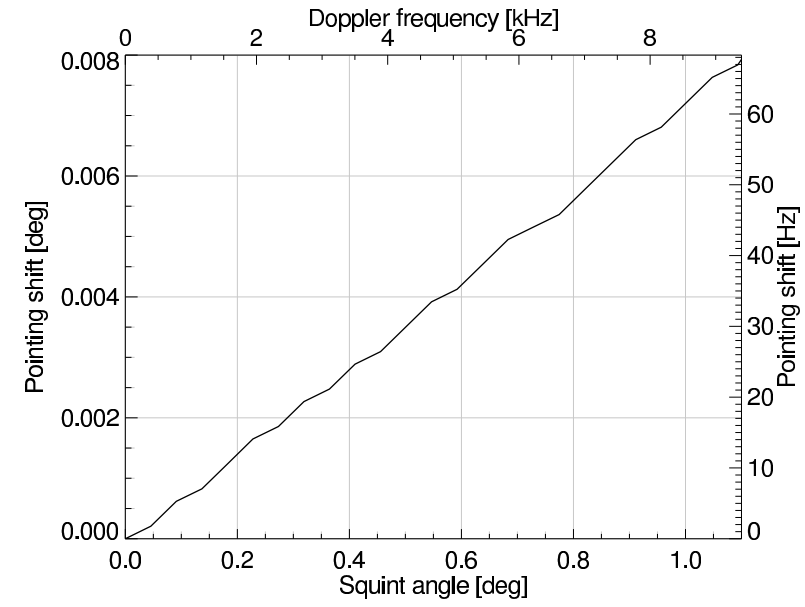

Fig. 4. Pointing shift due to the Element Pattern modulation effect for the TerraSAR-X system.

TABLE II

TERRASAR-X PARAMETERS

\begin{tabular}{lc}
\hline Wavelength $(\lambda)$ & $0.031 \mathrm{~m}$ \\
Orbit height & $514 \mathrm{~km}$ \\
Orbital repeat cycle & 11 days \\
Antenna dimension in azimuth $(L)$ & $4.784 \mathrm{~m}$ \\
Number of elements in azimuth & 12 \\
Number of azimuth beams & 249 \\
StripMap bandwidth $\left(\mathrm{PBW}_{0}\right)$ & $2765 \mathrm{~Hz}$ \\
\hline
\end{tabular}

The larger steering excursion of TOPS2+ with respect to TOPS or TOPS2 has some additional consequences related to the modulation effect of the Element Pattern (EP) of the antenna. The first one is related to a stronger Signal-to-Noise Ratio (SNR) variability over azimuth. This effect is further analyzed in section III-D and is taken into account for the performance evaluation in section IV.

The second effect of the element pattern modulation is the (squint-dependent) shift introduced to the position of the main lobe of the Directivity Pattern (DP) in azimuth direction. This has a (small) impact on the actual pointing direction and consequently, on the spectral separation between looks. The larger the squint angle, the larger the Doppler shift. In Fig. 4 a numerical evaluation of the pointing shift is shown for the range of squint angles of TOPS2+. The maximum pointing shift is equal to $0.008^{\circ}$ and corresponds to the maximum squint of $1.1^{\circ}$, or equivalently $68 \mathrm{~Hz}$ Doppler shift for a Doppler of about $9.4 \mathrm{kHz}$. For comparison, in case of TOPS2, the maximum squint angle is about $0.55^{\circ}$, equivalent to a maximum Doppler of $4.7 \mathrm{kHz}$, the maximum mis-pointing is approx. $0.004^{\circ}$, equivalent to about $32 \mathrm{~Hz}$. The Doppler shift relative to the Doppler frequency is approximately $0.7 \%$. Although the impact is small for interferometric applications, an effective antenna Doppler rate, $k_{\mathrm{rot}}^{\text {eff }}$, can be easily computed and employed during the SAR Processing.
B. Azimuth ambiguities, max. steering angle and PRF selection

The limiting factor to the largest steering is due to the level of the azimuth ambiguities, as a consequence of sampling the Doppler spectrum at a frequency PRF. The largest contribution to the azimuth ambiguities level is due to the grating lobe, therefore if we just consider it, the optimal PRF can be selected so that the digital frequency of the grating lobe is equal to 0.5 , i.e., the edge of the Doppler band. Following this strategy, a significant part of the energy from the grating lobe will fall outside the processing bandwidth. This condition can be written as:

$$
\frac{f_{\mathrm{GL}}}{\mathrm{PRF}_{k}}=\frac{1}{2}+k, k \in \mathbb{N},
$$

where $f_{\mathrm{GL}}$ is the Doppler frequency at which the maximum of the grating lobe is located and $k$ accounts for the periodicity of the spectrum of the sampled signal. Since the angular separation between the main lobe and the first grating lobe can be approximated for relative small angles by $\frac{\lambda}{d}$, being $d$ the distance between antenna elements in azimuth direction, the frequency of the grating lobe can be written as:

$$
f_{\mathrm{GL}} \approx \frac{2 v_{\mathrm{s}}}{\lambda}\left(\sin (\beta)+\sin \left(\frac{\lambda}{d}\right)\right),
$$

being $\beta$ the squint angle and $d$ the distance between elements of the array.

The set of PRFs which minimize the azimuth ambiguity from the first grating lobe is given by:

$$
\mathrm{PRF}_{k}=\frac{\frac{2 v_{\mathrm{s}}}{\lambda}\left(\sin \left(\beta_{\text {max }}\right)+\sin \left(\frac{\lambda}{d}\right)\right)}{k+\frac{1}{2}}, k \in \mathbb{N},
$$

where note that the maximum squint angle, $\beta_{\max }$, is employed, since the grating lobe reaches its highest level for the maximum steering.

Equation (15) provides an approximation for the optimal PRF, since it only accounts for the first grating lobe and is also inaccurate when the maximum sweeping angles increase.

A more precise value for the optimal PRF can be obtained by computing numerically the integrated Azimuth-Ambiguity-toSignal-Ratio (AASR) according to following expression:

$$
\mathrm{AASR} \approx \frac{\sum_{\substack{k=-\infty \\ k \neq 0}}^{\infty} \int_{-B_{\mathrm{a}} / 2}^{B_{\mathrm{a}} / 2} W\left(f_{\mathrm{d}}\right) \cdot G_{\mathrm{a}}^{2}\left(f_{d}+k \cdot P R F\right) \cdot \mathrm{d} f_{\mathrm{d}}}{\int_{-B_{\mathrm{a}} / 2}^{B_{\mathrm{a}} / 2} W\left(f_{\mathrm{d}}\right) \cdot G_{\mathrm{a}}^{2}\left(f_{\mathrm{d}}\right) \cdot \mathrm{d} f_{\mathrm{d}}},
$$

where $B_{\mathrm{a}}$ is the azimuth processing bandwidth, $W\left(f_{\mathrm{d}}\right)$ is the sidelobe suppression weighting function applied during processing, and $G_{\mathrm{a}}^{2}\left(f_{\mathrm{d}}\right)$ is the two-way far field azimuth antenna power pattern. Fig. 5 shows the result of the computation presented as a two-dimensional map for a range of 


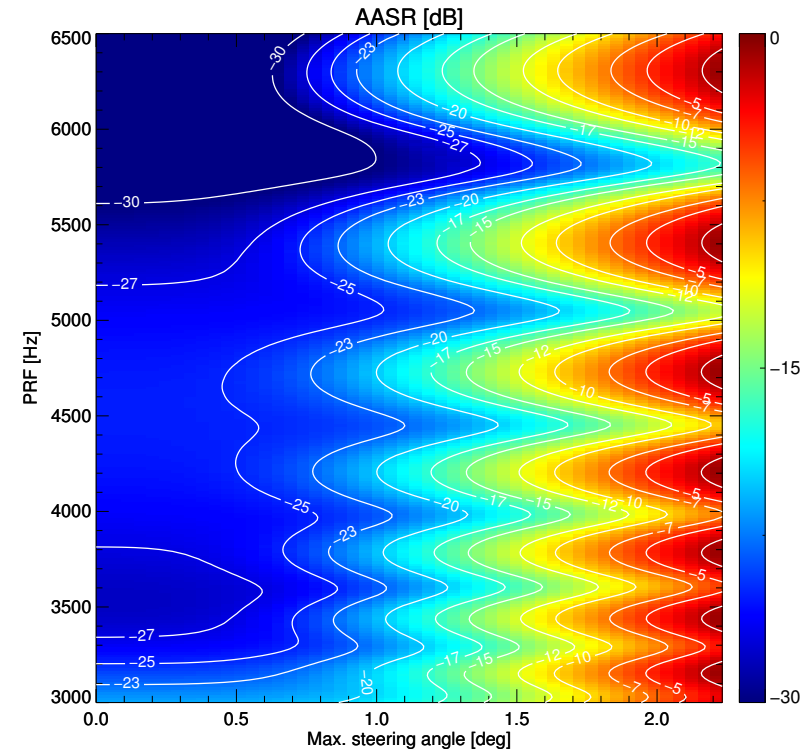

Fig. 5. Azimuth-Ambiguity-to-Signal Ratio (AASR) diagram for a set of PRFs and squint angles. It can be appreciated that, in general, the higher the squint angle, the higher the AASR level, however, the oscillating behavior of the AASR with the PRF indicates the possibility to select a set of optimal PRFs.

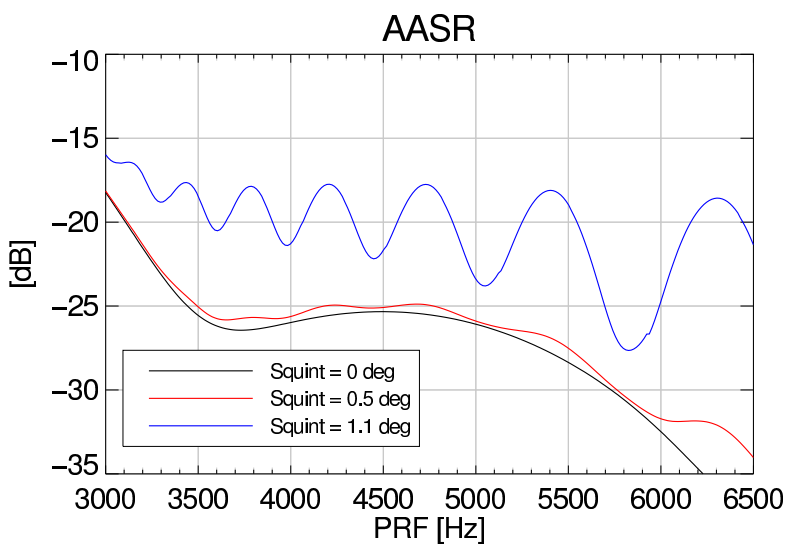

Fig. 6. AASR plot as a function of the PRF for the case of non squinted beam (StripMap), a squint of $0.5^{\circ}$ (TOPS or TOPS2) and a squint of $1.1^{\circ}$ (TOPS2+). The curves of StripMap and TOPS/TOPS2 present a very similar behavior and level, whereas the TOPS2+ curve presents a higher AASR and an oscillating behavior, having some minima for certain PRFs, in which the energy of the ambiguities is minimized. These sampling frequencies should be exploited for an optimal PRF selection.

PRFs and squint angles. A processing bandwidth of 2765 $\mathrm{Hz}$ has been considered and a generalized Hamming tapering window with $\alpha=0.6$ applied to the Doppler spectrum. Fig. 6 shows three profiles of the AASR for the case of no squint (StripMap-like), a squint $=0.5^{\circ}$ (TOPS or TOPS2) and a squint $=1.1^{\circ}$ (TOPS2 + ). There is an oscillating behavior of the AASR for TOPS2+ with the PRF, having several local minima in which the energy of the ambiguities is minimized. The selection of the PRF is, however, also restricted by the desired swath width $\left(W_{s}<\frac{c_{0}}{2 \cdot \mathrm{PRF}}\right)$ and the total Ambiguitiy-toSignal Ratio (ASR). In order to account for these parameters, analyses including the range ambiguities are provided in the next section.
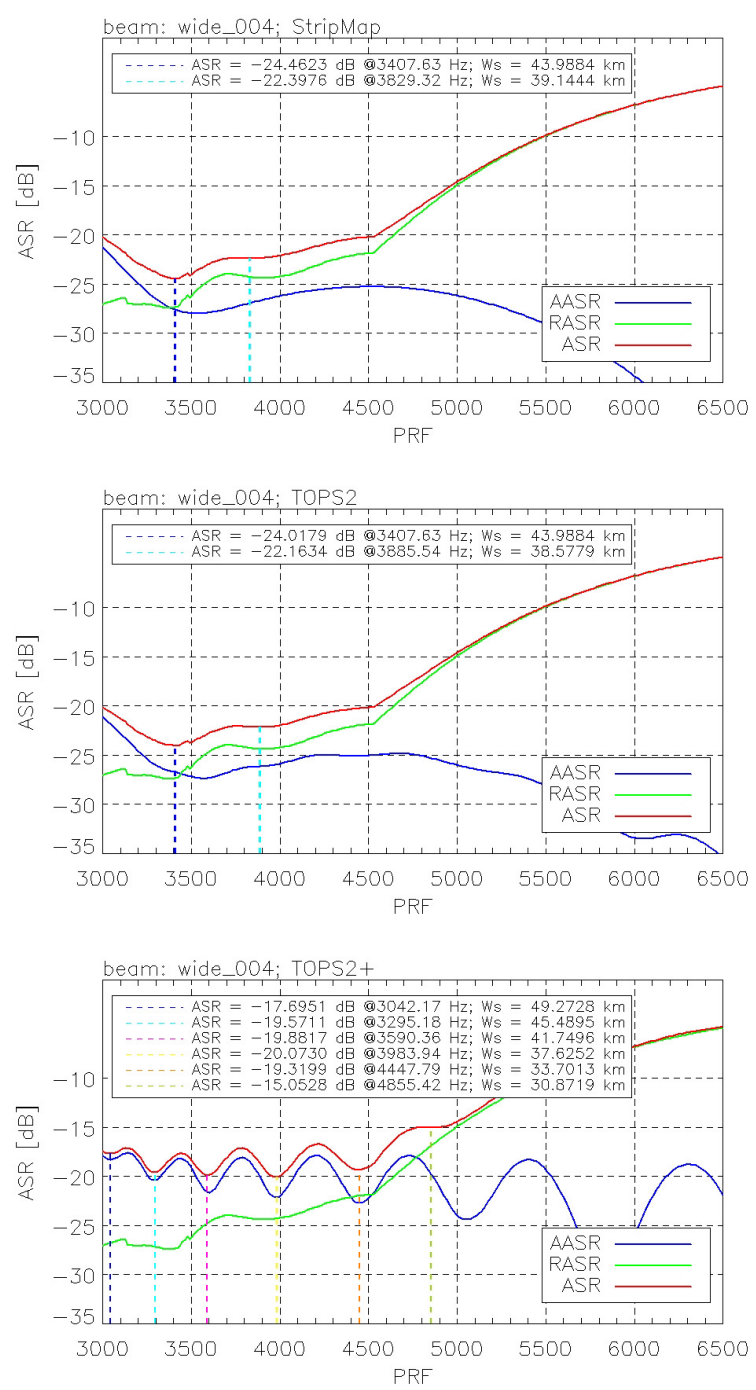

Fig. 7. Ambiguity-to-Signal Ratio in azimuth (AASR), elevation (RASR) and total (ASR), as a function of the PRF for the (top) StripMap mode, (middle) TOPS2 mode and for the (bottom) TOPS2+ mode. The TerraSAR-X beam wide004 (look angle at mid range $=30.3^{\circ}$ ) has been employed.

\section{Range ambiguities}

A second constraint to limit the sampling of the Doppler Spectrum with a high PRF is given by the range ambiguities [23]. The Range-Ambiguity-to-Signal-Ratio (RASR) can be obtained by considering the acquisition geometry, antenna pattern and scattering models. The optimal PRF in terms of ambiguities will be given by the one which minimizes the ASR, result of combining RASR and AASR.

Fig. 7 shows the curves of the RASR, AASR and ASR for the TerraSAR-X wide004 beam (look angle at mid range $=30.3^{\circ}$ ) in case of using StripMap (top) TOPS2 (middle) and TOPS2+ (bottom). The scatterer model for soil and rock from Ulaby [24] has been used for this computation. The location of the ASR local minima are indicated along with the ASR level. The difference of the AASR between StripMap and TOPS2 is almost negligible. The ASR curve for the TOPS2+ mode 
TABLE III

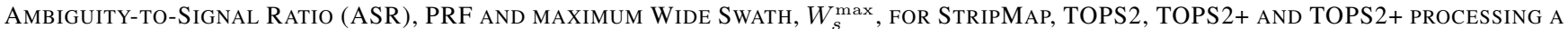
$70 \%$ OF THE AVAILABLE DOPPLER BANDWIDTH. THE LAST COLUMN INDICATES THE GAIN IN THE ASR WHEN PROCESSING THE REDUCED BANDWIDTH WITH RESPECT TO THE FULL BANDWIDTH FOR THE TOPS2+ MODE. THE AZIMUTH RESOLUTION OF THE FIRST SUBSWATH, $S S_{1}$, IS INDICATED.

\begin{tabular}{|c|c|c|c|c|c|c|c|c|c|c|c|c|c|}
\hline \multirow[t]{2}{*}{$n$} & \multicolumn{3}{|c|}{$\begin{array}{c}\text { StripMap } \\
\rho_{\text {az }}=3.3 m\end{array}$} & \multicolumn{3}{|c|}{$\begin{array}{c}\text { TOPS } 2 \\
\rho_{\mathrm{az}}^{S S_{1}}=16.6 m\end{array}$} & \multicolumn{3}{|c|}{$\begin{array}{c}\text { TOPS } 2+ \\
\rho_{\mathrm{az}}^{S S_{1}}=13.38 m \\
\end{array}$} & \multicolumn{4}{|c|}{$\begin{array}{c}\text { TOPS2+ }\left(\mathrm{PBW}=0.7 \mathrm{PBW}_{0}\right) \Delta \mathrm{ASR}_{\mathrm{TOPS} 2+}^{\mathrm{PBW}} \\
\rho_{\mathrm{az}}^{S S_{1}}=18.38 m\end{array}$} \\
\hline & ASR[d & PRF & $W_{\mathrm{s}}^{\max }[\mathrm{k}$ & $\mathrm{SR}[$ & $\mathrm{P}$ & $W_{\mathrm{s}}^{\max }[$ & B] & ] PRF & $W_{\mathrm{s}}^{\mathrm{m}}$ & 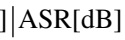 & RI & $W_{\mathrm{s}}^{\mathrm{m}}$ & {$[\mathrm{dB}]$} \\
\hline 001 & -27.91 & 3534.14 & 42.41 & -27.31 & 3562.25 & 42.08 & -21.59 & 3604.42 & 41.58 & -22.91 & 3604.42 & 41.58 & 1.32 \\
\hline 002 & -27.70 & 3520.08 & 42.58 & -27.11 & 3562.25 & 42.08 & -21.54 & 3604 & 41.58 & -22.84 & 3604.42 & 41.58 & 1.30 \\
\hline 003 & -27.91 & 353 & & & 3562.25 & 42.08 & & 360 & 4 & -22.91 & & 41 & 1.32 \\
\hline 004 & -24.46 & 3407.73 & 99 & .02 & 3407.63 & 43.99 & .07 & 398 & 37.63 & -21.07 & .12 & 45 & 1.00 \\
\hline 005 & -24.36 & 3491.97 & 42.93 & -24.02 & 3520.08 & 42.56 & -20.36 & 3590.36 & 41.75 & -21.33 & 3590.36 & 41.75 & 0.97 \\
\hline 006 & -27.59 & 3534.14 & 42.41 & -26.99 & 3562.25 & 42.08 & -22.02 & 3983.94 & 37.62 & -22.89 & 3983.94 & 37.62 & 0.87 \\
\hline 007 & -27.53 & 3562.25 & 42.08 & -26.99 & 3562.25 & 42.08 & -21.85 & 3983.94 & 37.62 & -22.78 & 3604.42 & 41.58 & 0.93 \\
\hline 008 & & 3674.70 & & & 3604.42 & & & 398 & & -21.81 & 3969.88 & 37.76 & 0.70 \\
\hline 009 & -24 & 3506.02 & & 41 & 3520.08 & 42 & 55 & 360 & 41.56 & -21.58 & 3590.36 & 41.75 & 1.03 \\
\hline 010 & -24.79 & 3449.80 & 43.45 & -24.32 & 3449.80 & 43.45 & -19.83 & 3590.36 & 41.75 & -21.34 & 3281.12 & 45.68 & 1.51 \\
\hline
\end{tabular}

shows larger deviations due to the oscillating behavior of the AASR with the PRF.

Table III summarizes the ambiguities parameters for StripMap TOPS2 and TOPS2+ for each TerraSAR-X wide beam. The ASR is indicated along with its corresponding PRF, which moreover establishes the largest coverage. The level of ASR is located, in average, at $-25.66 \mathrm{~dB}$ for the TOPS2 mode and at $-21.1 \mathrm{~dB}$ for TOPS2+. From these analyses, we conclude that TOPS2+ achieves a spectral separation, which is approximately twice as large as for TOPS2, at the expense of an ASR degradation of $4.5 \mathrm{~dB}$. The maximum swath width for TOPS $2+$ is marginally narrower than for TOPS 2 .

One option to reduce the azimuth ambiguities level consists in processing a narrower Doppler bandwidth, $B_{\text {a }}$, at the expense of azimuth resolution. For the sake of illustration, Table III includes the values of the ASR for the TOPS2+ mode when a $70 \%$ of the available bandwidth is processed, along with its gain with respect to a full processing bandwidth approach. The gain is, in average, around $1 \mathrm{~dB}$. If stringent requirements on the ASR are to be fulfilled and/or the azimuth resolution shall not be degraded, the maximum squint angle of TOPS2+ can be relaxed.

\section{Residual scalloping effect and combination of looks}

The major aim of the TOPS mode is to solve the problem of scalloping and azimuth varying ambiguities present in ScanSAR [5]. However, due to the element pattern modulation effect, some residual scalloping is still present. The larger the squint angles, the larger the scalloping effect. Fig. 8 shows the evolution of the normalized intensity during the acquisition of one burst for TOPS2 and TOPS2+, according to the steering rates from Table I. The horizontal axis indicates the azimuth time. TOPS 2 presents a maximum decay of $0.36 \mathrm{~dB}$, whereas TOPS2+ has a decay of $1.76 \mathrm{~dB}$.

We enumerate in the following the way to combine the looks. Since our case of interest corresponds to scenes over land, we assume an scenario characterized by distributed targets.

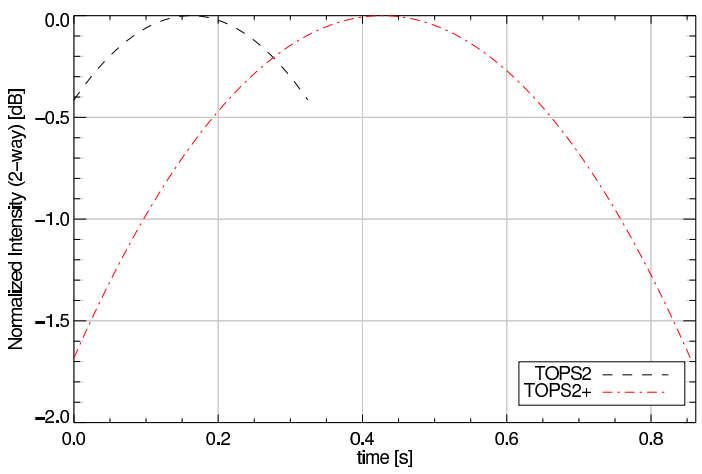

Fig. 8. Scalloping evaluation plot for TOPS2 and TOPS2+ modes. The normalized intensity is plotted during the acquisition of a single burst.

The speckle noise can be modeled as a complex signal, whose in-phase and quadrature components are independent and identically distributed zero-mean Gaussian variables. The benefit of combining the looks is three-fold:

(i) On the one hand, the differential interferogram between the interferograms of the looks provides an enhanced measurement of the azimuth shift, that can be considered a product by itself, but moreover aids to the across- and along-track decoupling procedure, supporting the spatial phase unwrapping of interferograms.

(ii) Secondly, the looks can be combined at the interferometric processing stage in order to obtain the 2-look interferogram, i.e. the coherent sum of the complex interferograms of each look. When performing this operation, the standard deviation of the interferometric phase is expected to be reduced by a factor $\sqrt{2}$.

(iii) The third benefit is the capability to reduce the speckle noise of the intensity image by applying an incoherent (spectral) multilooking, i.e., by averaging the intensities of each look.

Moreover since the TOPS mode varies progressively the line of sight, and having into account that odd and even bursts are interleaved in the time-frequency domain, according to the 

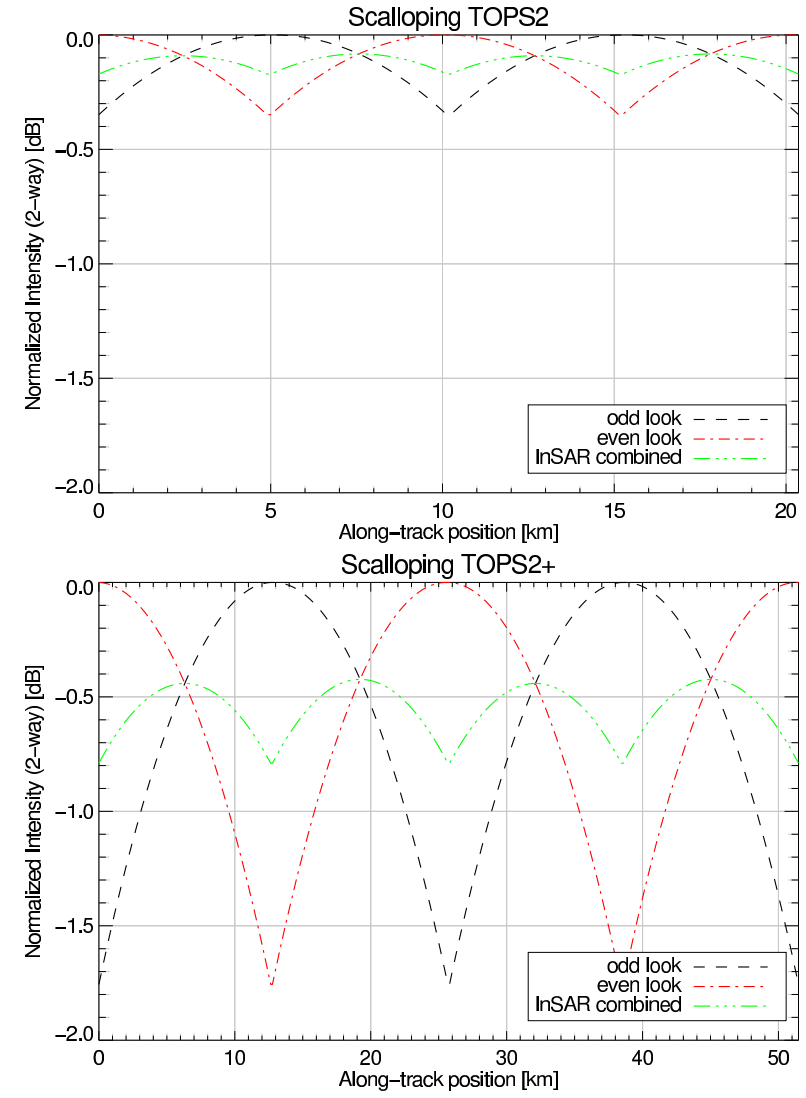

Fig. 9. Normalized intensity for the odd and even bursts and for the resulting interferogram when both looks are combined. The upper plot shows the values for TOPS 2 and the bottom lower plot for TOPS2+.

acquisition timeline (see Fig. 2), there is an additional benefit when combining both looks since SNR losses and azimuth ambiguities present, for a given target, different levels for each look, resulting in an equalization of both parameters.

Fig. 9 illustrates this fact for the SNR losses. The top part of the figure is related to the TOPS2 mode and shows the normalized intensity for two bursts of the odd looks and for two bursts of the even bursts according to their along-track ground position. If the interferograms of each look are combined, the resulting interferogram experiments an equalization, being the scalloping reduced from $0.36 \mathrm{~dB}$ to $0.07 \mathrm{~dB}$. The bottom part of the figure corresponds to TOPS2+, in this case the scalloping amplitude is reduced from $1.76 \mathrm{~dB}$ to $0.4 \mathrm{~dB}$, which is very close to the TOPS case.

\section{SENSITIVITY ANALYSIS}

The accuracy that can be obtained with the 2-look TOPS modes to derive azimuth displacements is provided in section IV-A and depends on several factors, being the spectral separation between looks, bandwidth and interferometric coherence the most relevant. In order to compare the performance of different modes, other factors like SNR losses and ambiguities have to be considered. The performance of the decoupling between along-track and line-of-sight directions is provided

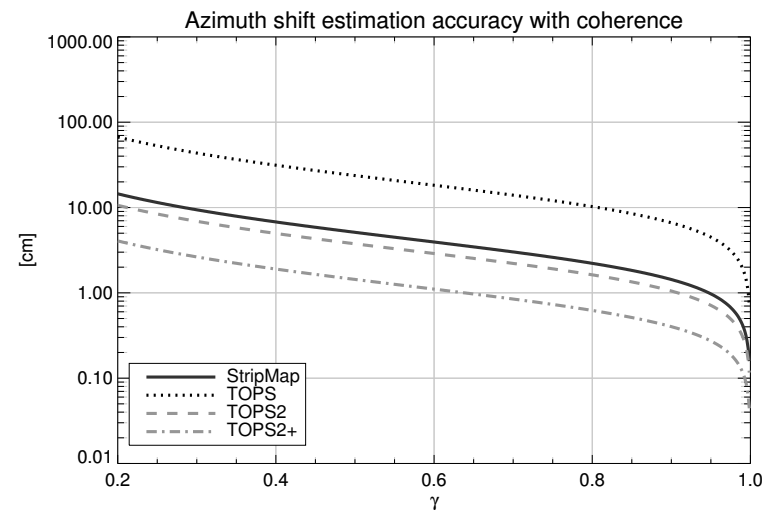

Fig. 10. Azimuth shift standard deviation for a StripMap, TOPS, TOPS2 and TOPS2+ system as a function of the coherence. The output product resolution has been set to $100 \mathrm{~m} \times 100 \mathrm{~m}$

in IV-B. Sections IV-C and IV-D include the consideration of tropospheric and ionospheric effects, respectively.

\section{A. Azimuth shift accuracy}

The estimation error of the azimuth shift, $\Delta x$, (in meter units) can be written combining (1) and the standard deviation of the differential phase between the interferograms of both looks, $\sigma_{\phi_{\mathrm{ESD}}}$. Assuming that the number of independent samples, $N$, is large, we can make use of the asymptotic phase noise expression of [25], obtaining:

$$
\sigma_{\Delta x}=\frac{\sigma_{\phi_{\mathrm{ESD}}}}{2 \pi \Delta f_{\mathrm{d}}} v_{\mathrm{g}}=\frac{1}{2 \pi \Delta f_{\mathrm{d}} \sqrt{N}} \frac{\sqrt{1-\gamma^{2}}}{\gamma} v_{\mathrm{g}}
$$

where $\gamma$ is the interferometric coherence.

The plot of the accuracies in the retrieval of the azimuth mutual shift between two images, for TOPS, TOPS2 and TOPS2+ with a maximum steering angle of $1.1^{\circ}$, is shown in Fig. 10, as a function of the coherence. The curves have been generated taking into account the spectral separation between looks, bandwidths, and establishing an output product resolution of $100 \mathrm{~m} \times 100 \mathrm{~m}$.

If we define the relative variance between each mode and the reference mode, StripMap (SM) as:

$$
\rho=10 \cdot \log _{10} \frac{\sigma_{\Delta x}^{2}}{\sigma_{\Delta \mathrm{x}}^{\mathrm{SM}} 2}=10 \cdot \log _{10} \frac{\left(\Delta f_{\mathrm{d}}^{\mathrm{SM}}\right)^{2} \cdot B_{\mathrm{T}}^{\mathrm{SM}}}{\Delta f_{\mathrm{d}}^{2} \cdot B_{\mathrm{T}}},
$$

we obtain that the performance of TOPS is $13.3 \mathrm{~dB}$ worse than the StripMap one, whereas the TOPS2 mode is $2.7 \mathrm{~dB}$ better. In the case of TOPS2+, with a maximum steering angle of $1.1^{\circ}$, the gain becomes $11 \mathrm{~dB}$. Since it can be very abstract for the reader to speak in terms of $\mathrm{dB}$ when comparing accuracies, we can equivalently say that the standard deviation of a TOPS mode is 4.6 times worse than for Stripmap. Regarding TOPS2 and TOPS2+, the standard deviations are 0.73 and 0.28 times the StripMap one, respectively. The gain of the TOPS2+ mode 

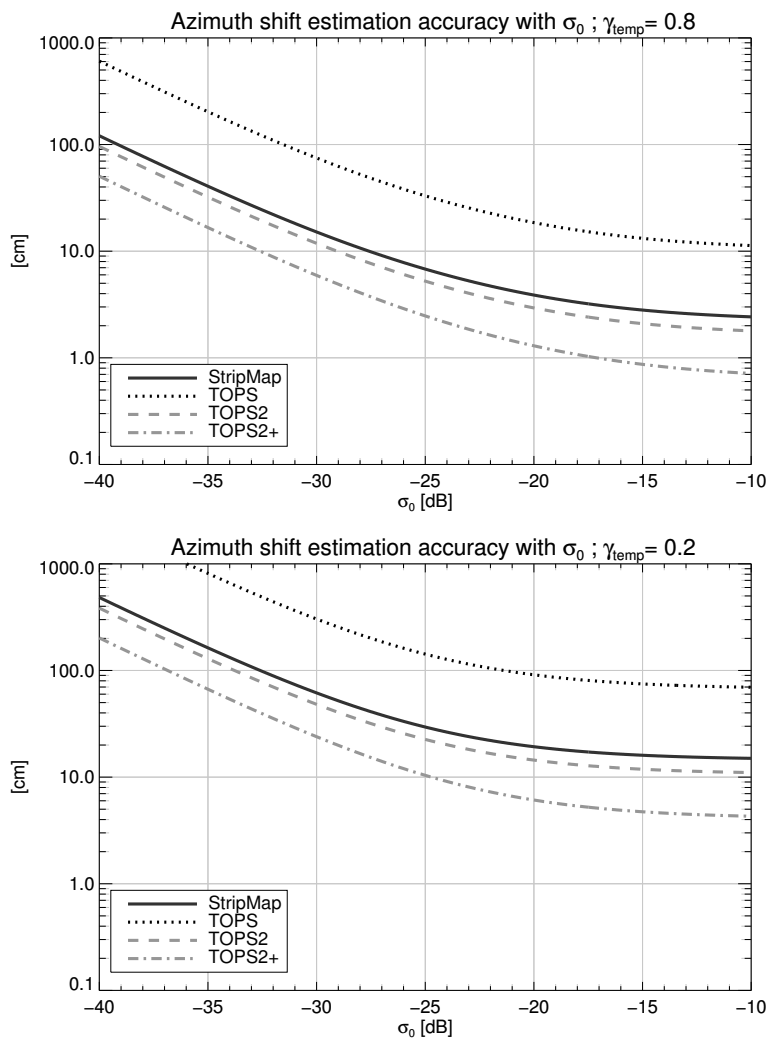

Fig. 11. Azimuth shift standard deviation for a a StripMap, TOPS, TOPS2 and TOPS2+ system for a common output product resolution of $100 \mathrm{~m} \times 100 \mathrm{~m}$ as a function of the backscattering coefficient. The upper plot corresponds to high temporal coherence (0.8), the lower plot to low temporal coherence (0.2). A Noise Equivalent Sigma Zero (NESZ) of $-25 \mathrm{~dB}$ has been assumed.

with respect to conventional TOPS is $24.3 \mathrm{~dB}$, which translates into a standard deviation 16.5 times smaller.

The interferometric coherence would, however, diverge among the modes, since each mode is unequally affected by ambiguities and SNR losses. In order to account for these effects, the accuracy analysis can be done as a function of the backscattering coefficient. Thus we model the total coherence, $\gamma$, as follows:

$$
\gamma=\gamma_{\mathrm{temp}} \cdot \gamma_{\mathrm{SNR}} \cdot \gamma_{\mathrm{amb}}
$$

where $\gamma_{\mathrm{temp}}$ is the temporal coherence and $\gamma_{\mathrm{SNR}}$ and $\gamma_{\mathrm{amb}}$ represent the error contribution due to a limited SNR and ambiguity ratio, respectively. The SNR can be written as:

$$
\mathrm{SNR}=\frac{\sigma_{0}}{10^{\frac{\left(\mathrm{NESZ}_{\mathrm{SNR}} \mathrm{Soss}\right)}{10}}},
$$

being $\sigma_{0}$ the normalized backscattering coefficient, NESZ the Noise Equivalent Sigma Zero level, in $\mathrm{dB}$, and $\mathrm{SNR}_{\text {loss }}$ the maximum losses in the SNR due to the modulation effect of the antenna element pattern when introducing a steering, also in $\mathrm{dB}$.
The limited SNR produces a coherence loss, being the resulting coherence [26]:

$$
\gamma_{\mathrm{SNR}}=\frac{1}{1+\mathrm{SNR}^{-1}}
$$

As detailed in Section III-D, the scalloping effect introduces a maximum SNR loss of $0.36 \mathrm{~dB}$ and $1.76 \mathrm{~dB}$ for TOPS2 and TOPS2+, respectively.

The error introduced by distributed ambiguities can be approximately modeled as Gaussian noise, whose contribution to the coherence loss is given by:

$$
\gamma_{\mathrm{amb}}=\frac{1}{1+\mathrm{AASR}} \cdot \frac{1}{1+\mathrm{RASR}},
$$

being AASR and RASR the azimuth and range ambiguity to signal ratios, respectively.

The coherence penalization due to ambiguities will be different among the swaths, in average, $\gamma_{\mathrm{amb}}>0.996$ for TOPS2 and $\gamma_{\mathrm{amb}}>0.989$ for TOPS2+.

Taking into account the mentioned losses, we obtain the performance curves of Fig. 11 as a function of the backscattering coefficient, where a NESZ of $-25 \mathrm{~dB}$ has been assumed. Since the dependence of the standard deviation with the coherence is not linear, we consider the case of high (0.8) and low (0.2) temporal coherences. For both cases it can be appreciated that the StripMap and TOPS2 curves get closer due to the residual scalloping and slightly larger azimuth ambiguities of TOPS2, however the latter is still better than StripMap. In spite of the larger SNR losses and greater azimuth ambiguities of TOPS2+, it still provides the best performance. When comparing the curves for high and low coherence, it can be concluded that the accuracy saturates with the backscattering coefficient more significantly for low temporal coherence than for high values. In section IV-C the effect of the turbulent troposphere is also taken into account, where the 2-D and a 3-D achievable performances are, as well, provided.

\section{B. Decoupling. Across-track phase accuracy}

The phase of the interferogram of each look, $\phi_{\text {look }} i$, contains, apart from the topographic phase, the contribution of the ground displacement vector, $\vec{g}$, measured in the line of sight (LOS) of each look, $\widehat{e}_{\text {los }} i$, which is given by [18]:

$$
\begin{aligned}
\phi_{\text {look } i} & =\frac{4 \pi}{\lambda}<\widehat{e}_{\text {los } i}, \vec{g}> \\
& =\phi_{\mathrm{XT} i}+\phi_{\mathrm{AT}} i, i=1,2,
\end{aligned}
$$

where $\phi_{\mathrm{XT} i}=\frac{4 \pi}{\lambda} \Delta R_{0}$ is the contribution to the interferometric phase in the across-track direction, being $\Delta R_{0}$ the projection of the ground displacement on the zero-Doppler plane; and $\phi_{\mathrm{AT} i}(t)=2 \pi \cdot f_{\mathrm{d} i}(t) \cdot \Delta t$ the additional phase due to the displacement in the along-track direction, $\Delta t$, being $f_{\mathrm{d} i}(t)$ the instantaneous Doppler frequency for each look, $i$.

A decoupling of the displacement in the across-track and along-track direction can be carried out by combining the 
phases of both looks. In the following, we analyze the phase noise in the interferometric phase after removing the contribution of the along-track deformation estimated with ESD. The phase noise related to the azimuth component of the deformation is given by

$$
\sigma_{\phi_{\mathrm{AT}}}(t)=2 \pi \cdot f_{\mathrm{d}}(t) \cdot \sigma_{\Delta t},
$$

where $\sigma_{\Delta t}$ is given by (17), after dividing by $v_{g}$. The worst case corresponds to the largest Doppler centroid, given at the burst edges. For a 2-look system, this maximum is approximately equal to the spectral separation between looks, i.e., $f_{\mathrm{d}}^{\max }(t)=\Delta f_{\mathrm{d}}$. Therefore, from (17) and (24), the largest phase noise can be written as:

$$
\sigma_{\phi_{\mathrm{AT}}}=\frac{1}{\sqrt{N_{\mathrm{ESD}}}} \frac{\sqrt{1-\gamma^{2}}}{\gamma},
$$

where $N_{\text {ESD }}$ corresponds to the effective number of looks to estimate the ESD phase.

The standard deviation of the across-track phase for each look can be written as:

$$
\begin{aligned}
\sigma_{\phi_{\mathrm{XT} i}} & =\sqrt{\sigma_{\phi_{\mathrm{look} i}}^{2}+\sigma_{\phi_{\mathrm{AT} i}}^{2}} \\
& =\sqrt{\frac{1}{2 N}+\frac{1}{N_{\mathrm{ESD}}} \frac{\sqrt{1-\gamma^{2}}}{\gamma}},
\end{aligned}
$$

where $N$ is the spatial multilooking factor of the interferograms of each look. After applying the same multilook to both looks and to the ESD phase, removing the along-track component to each look and averaging them, the standard deviation of the final across-track phase results in:

$$
\sigma_{\phi_{\mathrm{XT} 2-\mathrm{look}}}=\frac{\sigma_{\phi_{\mathrm{XT} i}}}{\sqrt{2}}=\sqrt{\frac{3}{2}} \sigma_{\phi_{\text {look } i}} .
$$

By increasing the ESD multilooking by a factor 2, i.e., $N_{\text {ESD }}=2 N$, the standard deviation of the across-track phase results in the standard deviation of the phase of each look, i.e., $\sigma_{\phi_{\mathrm{XT} 2-\mathrm{look}}}=\sigma_{\phi_{\text {look } i}}$. Note that this extra multilook reduces the resolution of the along-track deformation product by this same factor when compared to the across-track one, however this resolution loss does not play, in general, a big role.

\section{2-D and 3-D performance. Consideration of tropospheric effects}

In the following, the expected 2-D (line of sight and azimuth) and 3-D (combining acquisitions in ascending and in descending geometry) performances, including the influence of the turbulent troposphere, are presented.

The signal delay induced by the troposphere has been already characterized in the literature in the radar line of sight, when employing interferometric techniques [27]. The effect of the turbulent troposphere, also known as Atmospheric Phase Screen (APS), can be modeled, in that direction, as a noise source of a certain power, $\sigma_{\text {tropo,LOS }}^{2}$. When 2-look data are exploited to obtain the displacement in the azimuth direction, two repeat-pass interferograms are available, containing each of them almost the same tropospheric delay because of the small temporal and spatial baseline between looks. This fact has been already observed in [13] in the case of splitting the Doppler spectrum of StripMap images. In [28] a quantitative analysis for the case of simultaneous squinted SAR acquisitions with two or more platforms can be found.

In our particular scenario, we have a single platform employing 2-look modes in repeat-pass configuration. Since the acquisition of both looks is not simultaneous, an effective along-track baseline is present. From Table I we obtain a cycle time of $0.71 \mathrm{~s}$ for TOPS 2 and $1.77 \mathrm{~s}$ for TOPS2+, which is equivalent to an effective along-track baseline (at the height of the satellite) of $5.48 \mathrm{~km}$ and $13.95 \mathrm{~km}$, respectively. However, the relevant baseline is the one located at the height of the atmospheric boundary layer, i.e., the portion of the troposphere responsible for the turbu ence, which can be assumed to be at a height of approximately $1 \mathrm{~km}$. At this height the lines of sight of each look are separated by $6.78 \mathrm{~m}$ and $16.81 \mathrm{~m}$, respectively. The slightly different tropospheric delay experienced by the signal in both lines of sight is thus very small. The standard deviation of the tropospheric noise in the along-track direction (in meter units) is given by:

$$
\sigma_{\text {tropo }, \mathrm{AT}}=\frac{2 \sqrt{2 \tilde{R}(\Delta l)} v_{\mathrm{g}}}{\lambda \Delta f} \sigma_{\text {tropo,LOS }}
$$

where $\tilde{R}(\Delta l)$ is the normalized autocorrelation function of the turbulent troposphere evaluated at the along-track baseline between the lines of sight at the height of the turbulent troposphere, $\Delta l$. The autocorrelation function can be computed from the power spectrum of the turbulent troposphere, which can be modeled following the Kolmogorov's power law [28] [27]. By evaluating the (conservative) autocorrelation function assumed in [28] for the along-track baselines corresponding to TOPS 2 and TOPS2+, and considering a power of the turbulent troposphere in the line of sight, $\sigma_{\text {tropo,LOS }}^{2}=1 \mathrm{~cm}^{2}$, we obtain that the standard deviation of the tropospheric noise in the along-track direction is $1.41 \mathrm{~cm}$ for TOPS2, and $1.35 \mathrm{~cm}$ for TOPS2+. There is no degradation, in terms of tropospheric perturbations, of TOPS2+ with respect to TOPS2 when retrieving azimuth shifts. The smaller correlation between lines of sight of the latter is compensated with its larger spectral separation between looks, according to (28).

The effect of the turbulent troposphere in the along-track direction is therefore of similar order of magnitude than in the line of sight (for the assumed conservative autocorrelation function). This is due to a noise upscaling effect, as a consequence of the small angular diversity between the two looks. The performance for retrieving azimuth shifts in a real scenario implies the consideration of temporal decorrelation effects, where we will be able to determine which effect, temporal decorrelation or tropospheric noise, prevails. We make use of the Hybrid Cramér-Rao Bound (HCRB) for the crustal displacement field estimator provided in [29] and 
model the turbulent troposphere according to [28] with a power, $\sigma_{\text {tropo,LOS }}^{2}=1 \mathrm{~cm}^{2}$.

In order to provide the performances that are achieved when working with pairs and stacks of images, we employ an exponential decorrelation model [30] with a time constant of 40 days and a long-term coherence of 0.1 . The output product resolution has been set to $100 \mathrm{~m} \times 100 \mathrm{~m}$, which supposes 998 equivalent number of looks.

1) InSAR pairs: The top part of Fig. 12 shows the 2-D performance curves, i.e. range and along-track, in case of using pairs of images according to the temporal baseline for the TOPS2 (left) and TOPS2+ (right) modes. The achievable accuracy including tropospheric effects and without its consideration is shown at the top of the figure. The plots in the middle show the degradation of the accuracies due to the noise introduced by the troposphere. Regarding the performance in the along-track direction, we can see that for small temporal baselines (high coherence) the noise introduced by the troposphere dominates over temporal decorrelation effects. A few cycles later the degradation introduced by the troposphere becomes negligible, as the quotient between the accuracies with and without troposphere tends to 1 . From the plots of the degradation introduced by the troposphere, it seems that TOPS2+ is worse than TOPS2 in along-track, however it is a matter of the better sensitivity of TOPS2+ to displacements, which produces a larger ratio between tropospheric and temporal decorrelation effects. Observe that the final performance of TOPS2+ is better than TOPS2. Regarding the performance in range (across-track) direction, the level of noise introduced by the troposphere is much greater that the noise due to temporal decorrelation effects. The bottom part of the figure shows the 3-D performance when combining an ascending and a descending pair. Look angles of $30^{\circ}$ have been assumed for both geometries with orbit heading angles of $12^{\circ}$ and $168^{\circ}$, respectively. The improvement on the N-S direction is evident for TOPS2+. Moreover the performance in the vertical direction improves, benefited by the better estimation in along-track. We can see that the sensitivity is, in general, better for the E-W and vertical directions, however, for small temporal baselines the performances are closer for all three directions.

2) Time-series: In case of working with stacks of images, we are interested in obtaining the performance curves of the mean deformation velocities. The top part of Fig. 13 shows the accuracies in range (across-track) and along-track direction as a function of the number of images employed, assuming that an image is acquired each repetition cycle (11 days). As for the cases of using pairs, the curves including tropospheric effects and without its consideration are provided. The plots in the middle show the degradation of the accuracies due to the troposphere. Similar conclusions to the case of pairs of images can be drawn for the along-track direction: the noise introduced by the troposphere dominates over temporal decorrelation effects for very short stacks $(<10$ images). The effect of the troposphere becomes rapidly negligible when increasing the number of images (quotient $=1$ ). The tropospheric noise seems to be slightly larger for TOPS2+ than for TOPS2; this apparent behavior is not true, being the reason the better sensitivity of TOPS2+ to azimuth displacements, which produces that the tropospheric noise prevails slightly more over temporal decorrelation effects. Note that the final performance of TOPS2+ is better than TOPS2. For instance, TOPS2 achieves a standard deviation in the mean along-track velocity of $4 \mathrm{~mm} /$ month for a stack length of 30 images, whereas this can be achieved with just 13 images with TOPS2+. The bottom part of the figure shows the 3-D performance when combining ascending and descending stacks. Look angles of $30^{\circ}$ have been assumed for both geometries with orbit heading angles of $12^{\circ}$ and $168^{\circ}$, respectively. As for the case of using pairs, TOPS2+ presents an evident improvement for the N-S direction, as well as in the vertical direction. The sensitivity is, in general, better for the E-W and vertical directions, however for small stacks the performances are closer for all three directions.

\section{Consideration of ionospheric effects}

The interferometric compensation of ionospheric disturbances for burst-mode acquisitions has been tackled in [31], where a modification of the (range) split-spectrum method is proposed and applied to ScanSAR and TOPS data. For TOPS systems, gaps of the ionospheric phase screen appear in the azimuth direction due to the burst-mode acquisition nature and the high altitude of the ionosphere. In contrast, 2-look systems enable to reduce noticeably and even remove the gaps, which allows to carry out a better smoothing of the phase screen, specially relevant if high frequency variations are present.

The estimation noise of the split-spectrum technique imposes limitations to the achievable spatial resolution, allowing only to retrieve large-scale ionospheric variations. A powerful technique, that enables the estimation of the gradient of the ionospheric differential delay along azimuth, consists in calculating the mutual azimuth shifts between the interferometric images, as suggested in [32]. Since the 2-look TOPS modes provide an enhanced sensitivity to the azimuth shift, small-scale variations along azimuth can be retrieved. Therefore, joint estimations from split-spectrum and azimuth shifts are possible, without any limitation (in terms of data gaps or degraded sensitivity), as, e.g., applied in [33] to StripMap acquisitions.

\section{Methodology}

In the following, the methodology for the interferometric processing and exploitation of 2-look data is provided. Section V-A focuses on the the interferometric processing of pairs of images, combination of looks and retrieval of azimuth shifts, whereas section V-B provides the procedure to retrieve mean velocities with stacks.

\section{A. InSAR pairs}

The interferometric processing algorithms for TOPS data can be found, for instance, in [17] [34] [35], where a coregistration 

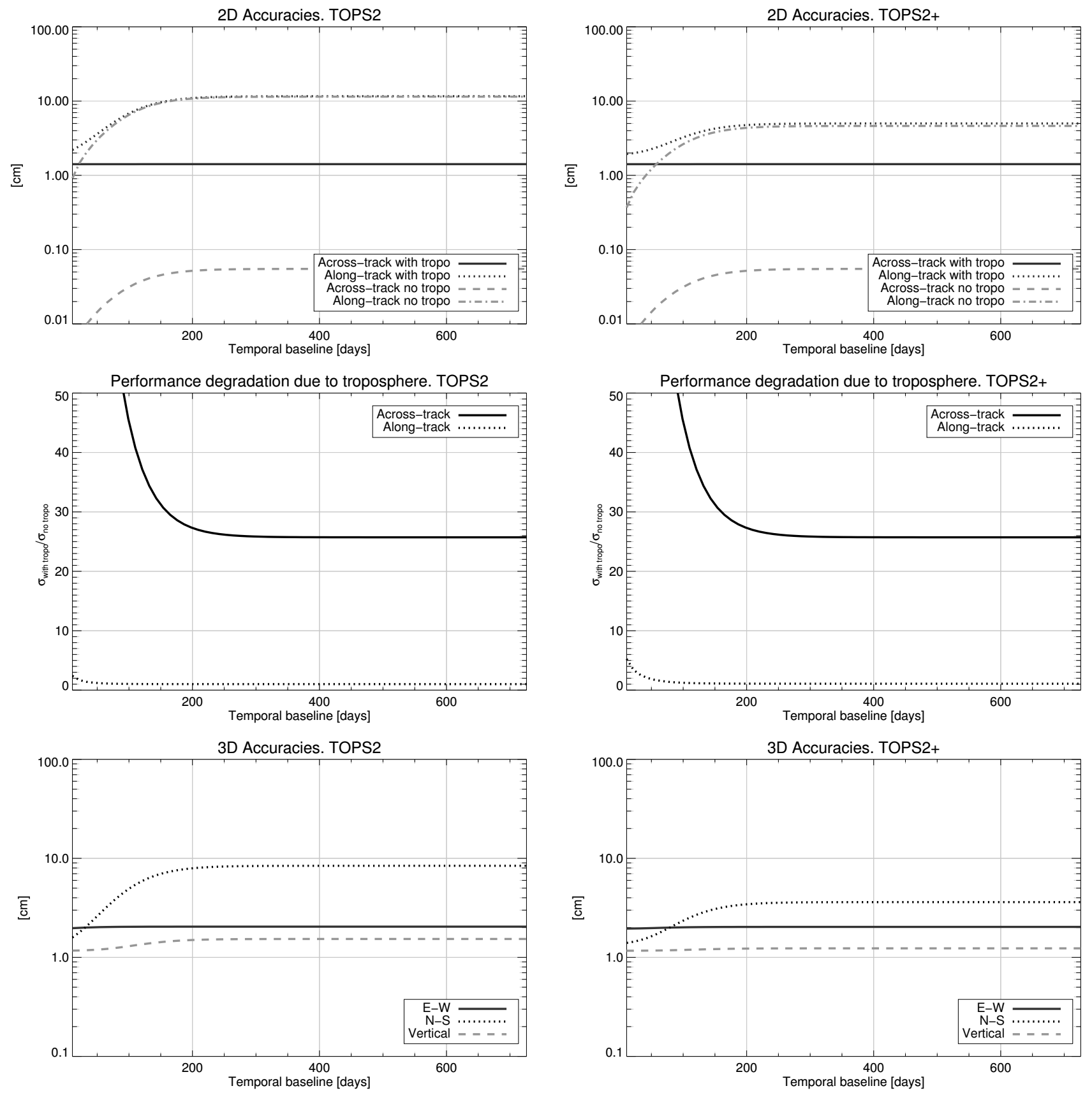

Fig. 12. The upper plots show the standard deviation of the shift measurement that can be retrieved in the line of sight and in the azimuth direction with an interferometric pair as a function of the temporal baseline, with and without considering the turbulent troposphere. The plots in the middle show the quotient of the accuracies in the retrieval of the shifts considering the troposphere and without its consideration. The effect of the troposphere is negligible in the along-track direction (quotient=1), except for very high coherences (small temporal baseline) where the tropospheric noise dominates. The output product resolution has been set to $100 \mathrm{~m} \times 100 \mathrm{~m}$, resulting in an equivalent number of looks of 998. The lower plots show the expected 3-D performance when combining two interferometric pairs with ascending and descending geometry with look angles of $30^{\circ}$ and orbit heading angles of $12^{\circ}$ and $168^{\circ}$. Left column refers to TOPS2, whereas right column to TOPS2+.

strategy, based on a geometric coregistration followed by ESD applied at the bursts overlap areas can correct for constant azimuth misregistration due to orbital or timing errors. Additional processing aspects, as the necessity to perform deramping and reramping operations for the spectral shift filter and resampling operations have been outlined. Fig. 14 shows the processing block diagram at burst-level, where a geometrical coregistration is done using precise orbital information and an external DEM following the procedure described in [36]. Spectral shift filtering can be applied optionally afterwards, specially important if large perpendicular baselines are expected.

The exploitation of both looks consists, in the first place, in calculating the map of local azimuth shifts by generating the differential interferogram between the interferograms of both 

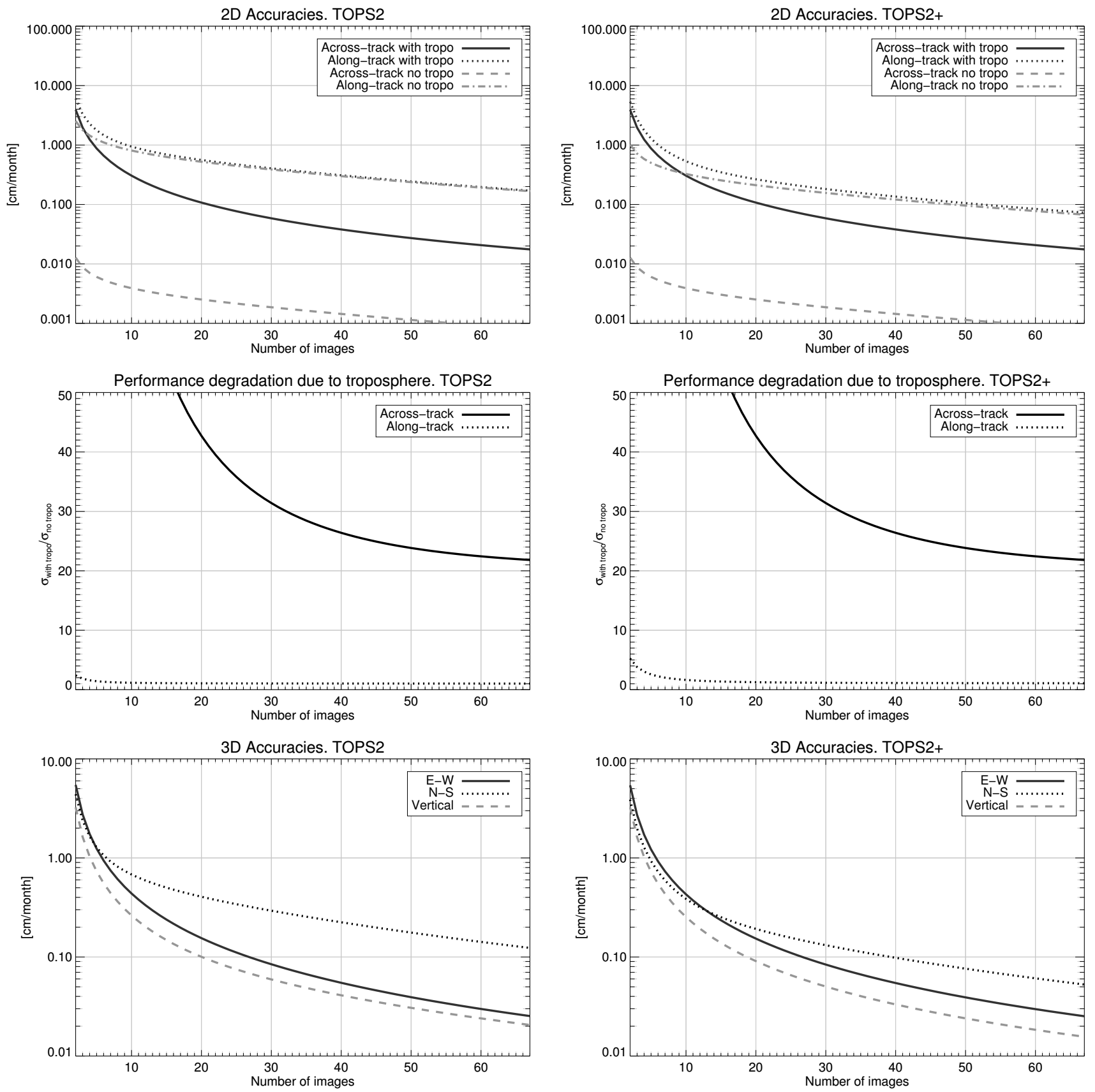

Fig. 13. The upper plots show the standard deviation of the shift measurement that can be retrieved in the line of sight and in the azimuth direction with a stack of images as a function of the number of acquisitions with and without the consideration of the turbulent troposphere, assuming that an image is acquired every 11 days. The plots in the middle show the degradation of the accuracies due to the troposphere. The tropospheric noise in the along-track direction is negligible (quotient=1), except for very short stacks, where tropospheric effects dominate over temporal decorrelation. TOPS2 achieves a standard deviation in the mean along-track velocity of $4 \mathrm{~mm} / \mathrm{month}$ for a stack length of 30 images, whereas this can be achieved with just 13 images with TOPS2+. The bottom part of the figure shows the 3-D performance when combining ascending and descending stacks. Look angles of $30^{\circ}$ have been assumed for both geometries with orbit heading angles of $12^{\circ}$ and $168^{\circ}$, respectively. Left column refers to TOPS2, whereas right column to TOPS $2+$.

looks (Int. look1 and Int. look2). The phase of the differential interferogram, $\phi_{\mathrm{ESD}}$, can be therefore written as:

$$
\phi_{\mathrm{ESD}}=\arg \left\{\left(m_{i} \cdot s_{i}^{*}\right) \cdot\left(m_{i+1} \cdot s_{i+1}^{*}\right)^{*}\right\},
$$

where $m_{\mathrm{i}}$ and $s_{\mathrm{i}}$ refer to the $\mathrm{i}^{\text {th }}$ master and slave complex bursts, respectively, and $m_{\mathrm{i}+1}$ and $s_{\mathrm{i}+1}$ refer to the $(i+1)^{\text {th }}$ master and slave bursts. Given a desired output product resolution, spatial multilooking can be applied to the InSAR phases / ESD phase. A small multilooking of the interferograms prior to the calculation of the differential interferogram (so-called early-multilooking) increases the estimation efficiency [37].

As mentioned before, the differential phase between both looks is affected by wrapping effects. Thus an unwrapping of 


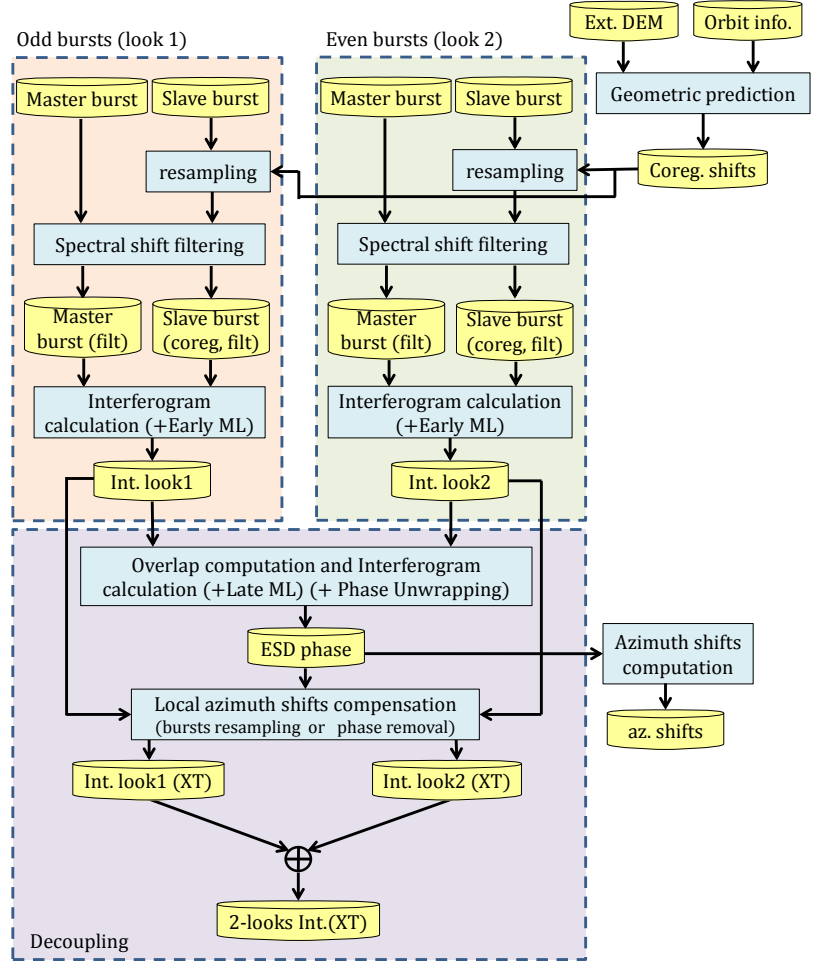

Fig. 14. Burst-level interferometric processing combining both looks. The slave bursts of booth looks are in first instance coregistered employing precise orbital information and an external DEM. Resampling and spectral shift filtering are performed considering the signal spectral properties, i.e. deramping and reramping operations are necessary (not shown in diagram). Afterwards the interferograms corresponding to each look are generated. The contribution to the phase of these interferograms is due to the projection of the ground displacement onto the line of sight of each look. The ESD phase can be computed by calculating the overlapping area between looks and forming the differential interferogram, applying the late multilooking; the ESD phase has to be unwrapped if shifts larger than the ambiguity band are expected. In order to obtain the 2-look interferogram, the ESD phase is used to decouple the displacement in across and along-track direction in order to obtain an interferogram which is sensitive to the displacement in the across-track direction (XT). The latter interferograms are averaged to obtain the 2-look across-track interferogram.

the differential phase becomes, in general, mandatory, when working with pairs. The azimuth shifts map, $\Delta t(r, x)$, can be retrieved according to:

$$
\Delta t(r, x)=\frac{\Gamma\left(\phi_{\mathrm{ESD}}(r, x)\right)}{2 \pi \Delta f_{d}(r)},
$$

where $\Gamma(\cdot)$ is the phase unwrapping operator.

For the generation of the 2-look interferogram we take advantage of the local azimuth shifts retrieved from the ESD phase. Its use makes possible the decoupling of the deformation in across-track and along-track direction, as analyzed in section IV-B. The removal of the phase due to azimuth displacement, $\phi_{\mathrm{AT}}$, consists in converting the ESD phase into azimuth shifts according to (30) and to resample again the slave bursts. If the azimuth shifts are small compared to the azimuth resolution, as a rule of thumb below $1 / 10^{\text {th }}$ to avoid degrading the interferometric coherence [38], the phase due to the azimuth displacements can be directly subtracted from the interferograms of each look. This phase can be calculated as:

$$
\phi_{\mathrm{AT}}(r, x)=\frac{f_{\mathrm{d}}(r, x)}{\Delta f_{\mathrm{d}}(r)} \Gamma\left(\phi_{\mathrm{ESD}}(r, x)\right) .
$$

The result are two interferograms, Int. look1 (XT) and Int. look2 (XT), whose phases are sensitive to the across-track direction. The interferograms of each look can now be coherently averaged in order to obtain the 2-look (across-track) interferogram. An additional multilooking could be applied at this stage according to the desired product resolution.

The suggested approach for the decoupling relies on a correct phase unwrapping of the ESD phase. The ESD phase is neither affected by residual topography nor turbulent troposphere, it suffers just from temporal decorrelation, which is, however, important for large temporal baselines and/or over vegetated areas.

In case the decoupling is not performed, the effective line of sight of the 2-look interferogram, resulting from the combination of the original interferograms of each look (Int. look1,2), presents a (time-varying) squint, $\beta_{2-\text { look }}(t)$, given by:

$$
\beta_{2-\text { look }}(t)=-\arcsin \left(\frac{\lambda}{4 v_{\mathrm{s}}}\left(f_{\mathrm{d}}^{\text {look1 }}(t)+f_{\mathrm{d}}^{\text {look2}}(t)\right)\right),
$$

where $f_{\mathrm{d}}^{\text {look1 }}(t)$ and $f_{\mathrm{d}}^{\text {look2}}(t)$ are the Doppler Centroid frequencies of the first and second look, respectively.

\section{B. Time-series}

Time-Series analysis of image stacks allows the retrieval of the temporal evolution of the deformation, where its mean velocity is usually obtained as a first step of the processing. In the case of 2-look systems, two equivalent approaches are possible in order to retrieve the along- and across-track deformation velocities, as expounded in the following.

From a geometrical point of view, we could retrieve the mean azimuth velocity by performing conventional independent time-series processings of each look, obtaining the mean velocities in the lines of sight of each of them. The mean azimuth velocity, $\widehat{v_{\mathrm{a}}}$, can be obtained by making the difference between the mean velocity for each look, $v_{\text {look } 1,2}$, and scaling the result according to:

$$
\widehat{v_{\mathrm{a}}} \approx \frac{2 \cdot v_{\mathrm{g}}}{\lambda \cdot \Delta f_{\mathrm{d}}} \cdot\left(v_{\text {look } 1}-v_{\text {look } 2}\right) .
$$

Since the estimation of the mean deformation velocity is performed independently for each look, the processing will have to cope with the presence of a higher noise due to the turbulent troposphere.

A second option consists in retrieving the azimuth mean velocity employing directly the ESD phases. The ESD phases can be computed from each pair of (looks) interferograms 
formed between a common selected master acquisition and the remaining slave images or jointly by calculating the ESD phases among all possible combination of acquisitions and using the Phase Linking algorithm [39]. The latter option provides a better estimation since the complete covariance matrix is exploited.

Provided that we have a stack of geometrically-coregistered acquisitions to a common master image, we can compute the ESD phases between each slave image and master. The mean azimuth velocity can be retrieved for each multilooked pixel by exploiting the following periodogram:

$$
\widehat{v_{\mathrm{a}}}=\underset{v_{\mathrm{a}}}{\arg \max }\left[\Re\left\{\sum_{i} e^{j\left(\phi_{\mathrm{ESD}}[i]-2 \pi \Delta f_{\mathrm{d}} v_{\mathrm{a}} T[i] v_{g}[i]\right)}\right\}\right],
$$

where $\arg \max _{v_{\mathrm{a}}}\{\cdot\}$ stands for the argument of the maximum (mean azimuth velocity, $v_{\mathrm{a}}$, for which the function attains its largest value). $\phi_{\mathrm{ESD}}[i]$ is the temporal array of ESD phases of each (master-slave) interferogram, $i$, for each multilooked pixel and $T[i]$ are the temporal baselines of each interferogram. Note that the dependency of $\Delta f_{\mathrm{d}}$ and $v_{\mathrm{g}}$ with range has been omitted for simplicity.

Working with the ESD phases directly instead of with each look independently has a major advantage, namely, the phases are autocalibrated, i.e., systematic effects, like residual topography, most of the baseline errors and tropospheric noise cancel out, being not necessary to establish a reference point to align the ESD interferograms. In scenarios with low subsidence rates, it can also be assumed that the phases due to ground motion are also smaller than the ambiguity band. Therefore (34) can be applied directly to each sample independently without having to exploit arcs between points as usually done when processing time series [40].

\section{Demonstration With TERraSAR-X: EXPERIMENTAL RESULTS}

Experimental TOPS2 acquisitions have been performed over different sites to demonstrate the applicability of the mode for the retrieval of large azimuth displacements employing pairs of images, and of mean deformation velocities by exploiting time series. The first example, with pairs of images, corresponds to the retrieval of the azimuth shifts on the Petermann glacier, located in North-West Greenland, with a main orientation to the N-S direction.

The second example, employing time-series, corresponds to the monitoring of post-seismic ground deformation after the 2013 Balochistan earthquake, in Pakistan.

\section{A. The Petermann glacier}

With its $80 \mathrm{~km}$ long and $16 \mathrm{~km}$ width, the Petermann glacier is the largest floating glacier in the Northern Hemisphere. It connects the Greenland ice sheet to the Arctic Ocean. Its study is of great interest among the glaciological community to determine how fast ice is being discharged to the seas. The
TABLE IV

MEAN DOPPLER FREQUENCY DIFFERENCES BETWEEN LOOKS AND CORRESPONDING ESD AMBIGUITY BAND. TOPS2 ACQUISITION WITH FOUR SUBSWATHS OVER PETERMANN GLACIER, GREENLAND.

\begin{tabular}{lcccc}
\hline & SS1 & SS2 & SS3 & SS4 \\
\hline$\left\langle\Delta f_{\mathrm{d}}^{\text {ovl }}\right\rangle[\mathrm{Hz}]$ & 3182 & 3127 & 3039 & 2979 \\
Ambiguity band $[\mathrm{m}]$ & \pm 1.11 & \pm 1.13 & \pm 1.16 & \pm 1.18 \\
\hline
\end{tabular}

Petermann glacier represents moreover a challenging scenario for our demonstration due to the heterogeneous motion gradient present [41]. A pair of TerraSAR-X TOPS2 images have been acquired on 16/10/2015 and 27/10/2015. The data takes are composed by four subswaths and 122 bursts (with a length of approx. $10 \mathrm{~km}$ each). The range coverage is $100 \mathrm{~km}$, with a total scene length of $500 \mathrm{~km}$, being the azimuth resolution $40 \mathrm{~m}$. Fig. 15 shows an overview of the obtained results, the horizontal direction corresponds to along-track. From the reflectivity (a), it can be seen that the ice flows from left to right, where the ice tongue can be distinguished. After a coregistration has been performed, the coherence (b), and InSAR phase (c) can be obtained. It can be appreciated that due to the high glacier velocity, the coherence is low on the ice tongue. Our focus is outside these areas, since even if there were enough coherence, there is no justification to apply interferometry due to very high fringe frequency. For such areas, amplitude-based techniques, e.g. cross-correlation, immune to wrapping effects are more adequate. The parts of the glacier with a slower displacement rate present an acceptable coherence and moderate velocities. A Greenland DEM obtained from the Ice Mapping Project (GIMP) [42] has been employed to subtract topographic fringes. On the left part of the InSAR phase (c), phase discontinuities due to glacier displacement can be observed. The aim is to remove the phase discontinuities by applying an accurate local azimuth coregistration by exploiting both looks, as expounded in section V-A. The combination of the InSAR phases from both looks allows the computation of the ESD phase. Table IV shows the spectral separation between looks and the ambiguity bands for each subswath, which is around $1 \mathrm{~m}$. Since larger movements in the azimuth direction are expected, the ESD phase (d) has to be unwrapped. We would expect to obtain a smoother ESD phase, however phase discontinuities are to be found at the burst interfaces indicating that there are ionospheric perturbations due to the high solar activity occurred in 2015, strong enough to be sensed at X band. An estimation of the ionospheric contribution employing, e.g., split bandwidth techniques [31] in order to separate displacement from ionospheric disturbances is out of the scope of our evaluation.

Once the azimuth shifts have been estimated, they are removed from the interferograms of each look by using (31) in order to retrieve the across-track deformation for each look. The phase of the average of both interferograms is shown in Fig. 15 (e), which is free of phase discontinuities. 


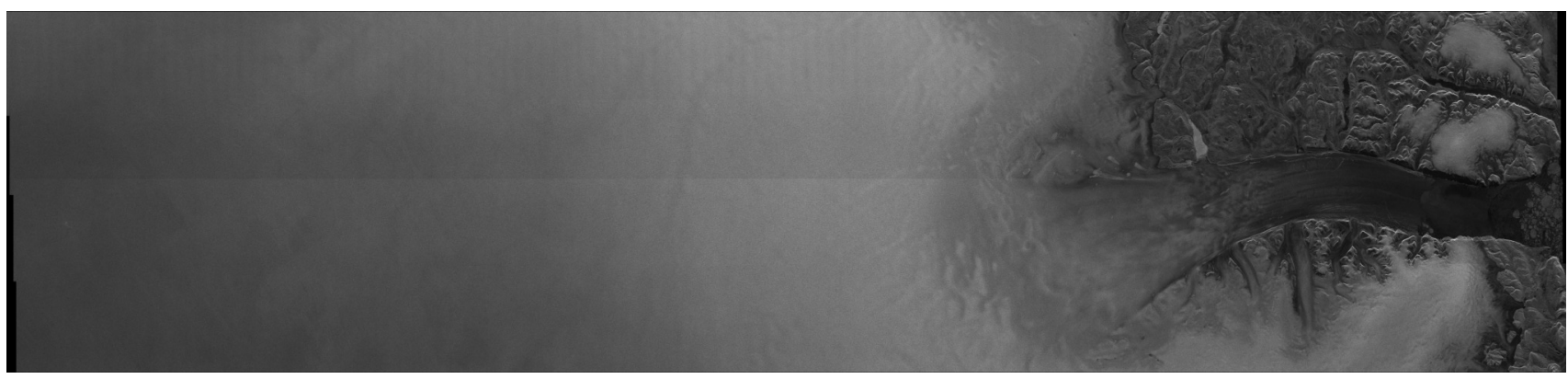

(a)

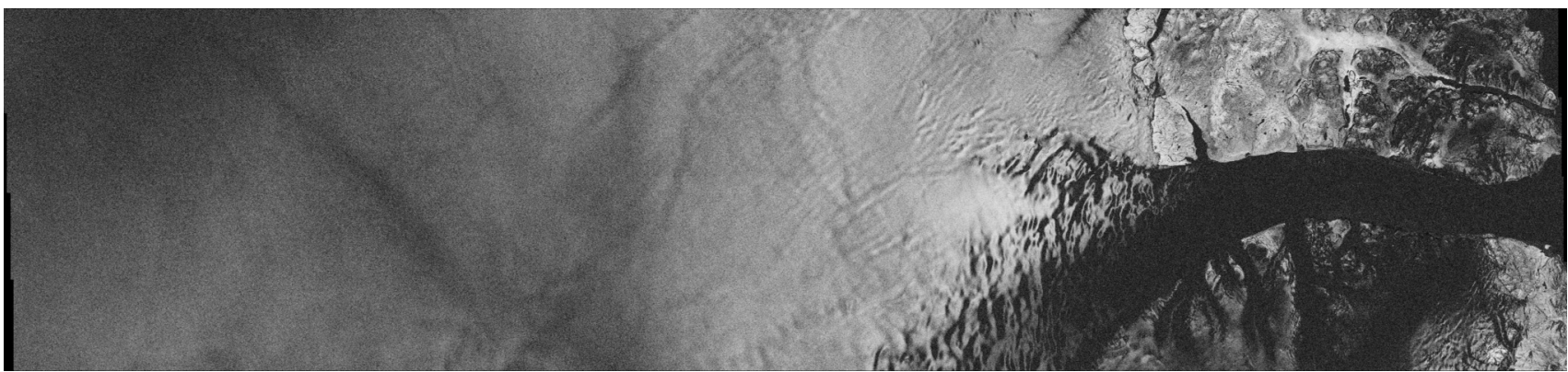

(b)

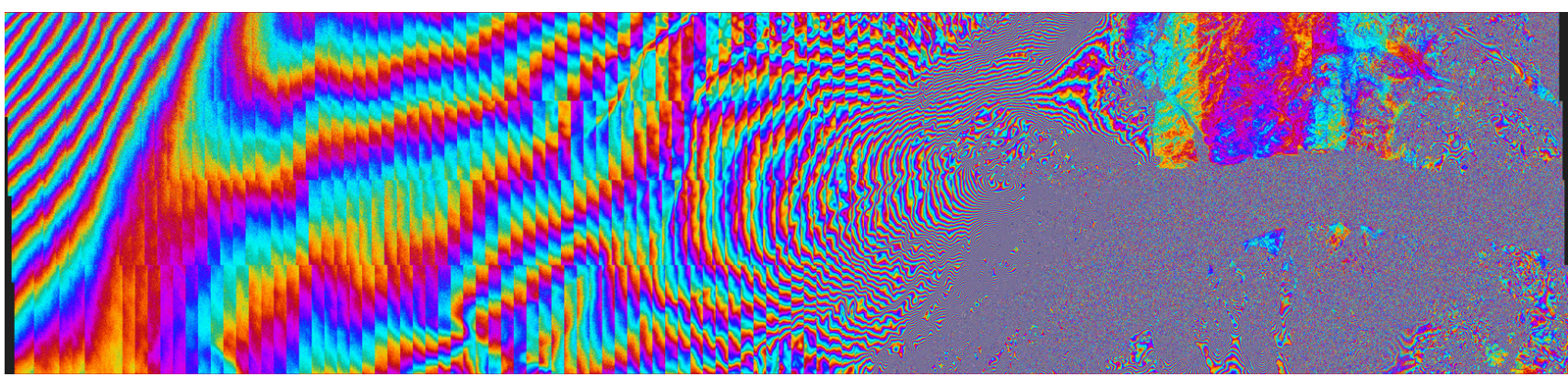

(c)

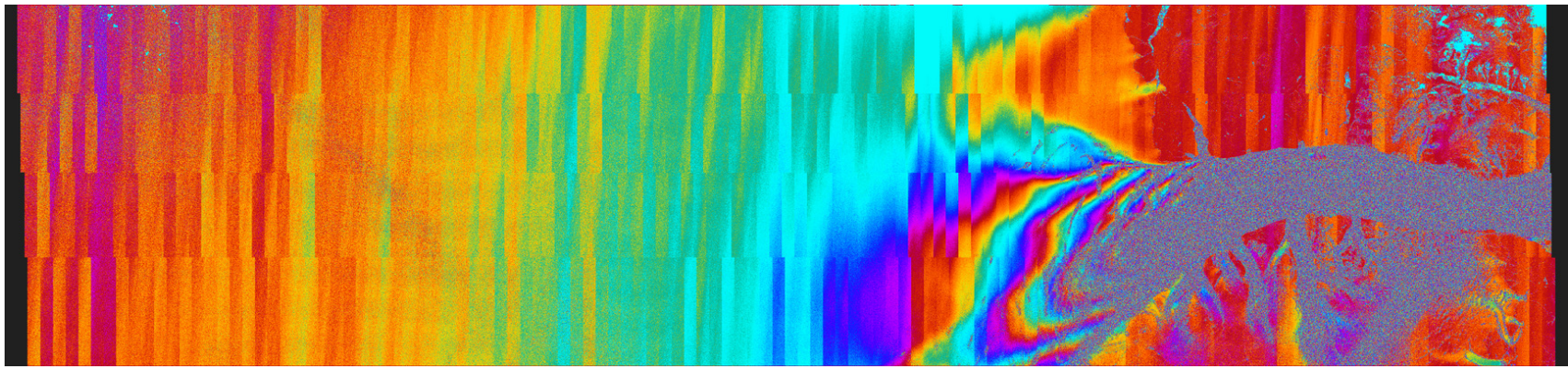

(d)

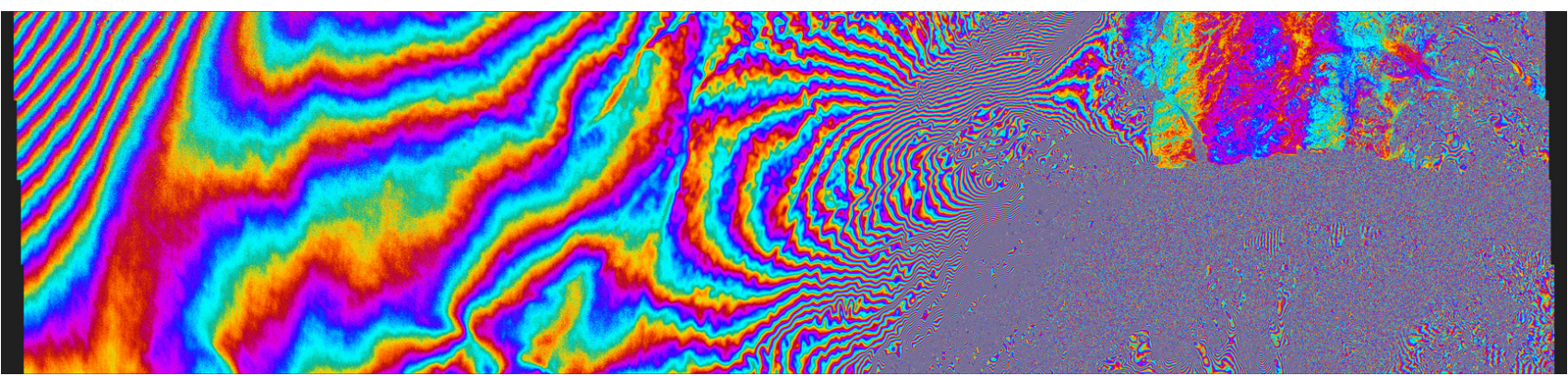

(e)

Fig. 15. Interferometric TOPS2 results over Petermann site corresponding to the pair 16/10/2015-27/10/2015. The acquisition covers an area of $100 \mathrm{~km} \times 500 \mathrm{~km}$ and employs four subswaths with an azimuth resolution of $40 \mathrm{~m}$. (a) Reflectivity, (b) coherence, (c) interferometric phase after removing the topography, (d) ESD phase proportional to the along-track motion, and (e) interferometric phase obtained after removing the along-track component of the motion by exploiting the 2 looks. The GIMP DEM has been used to remove the topography contribution to the phase. The phases are scaled between $\pm 180^{\circ}$. Range is vertical and azimuth is horizontal. 


\section{B. Post-seismic deformation after the $2013 M_{w} 7.7$ Balochistan earthquake}

In late September 2013 two main Earthquakes took place in Balochistan, South-West of Pakistan. The main shock had a magnitude of 7.7, while a $\mathrm{M}_{\mathrm{w}} 6.8$ aftershock occurred some days after. The co-seismic events have been analyzed using ground surface deformation measured from sub-pixel correlation of Landsat-8 images, combined with back-projection and finite source modeling of teleseismic waves [43]. Radar imagery was additionally used in [44]. The tectonics of southern and central Pakistan reflect a complex plate boundary where the India plate slides northward relative to the Eurasia plate in the east, and the Arabia plate subducts northward beneath the Eurasia plate in the Makran. These motions typically result in N-S to Northeast-Southwest strike-slip motion. After the 2013 shocks, post-seismic deformation close to the 2013 Epicenter location is expected, with displacements in N-S to NorthEast-SouthWest.

Two stacks of experimental TerraSAR-X TOPS2 acquisitions, in ascending and descending geometry, have been acquired from April 2016 until April 2018. The acquisitions have two subswaths and cover an extension of $85 \mathrm{~km} \times 265 \mathrm{~km}$ with $17 \mathrm{~m}$ azimuth resolution. Table $\mathrm{V}$ details the spectral separation between looks and the ambiguity bands for each subswath. We have analyzed two years of acquisitions in descending geometry with a total of 67 images. In ascending geometry a total of 53 images were successfully acquired in the same time interval. The top part of Fig. 17 shows the distribution of the perpendicular and temporal baselines for both, where note that acquisitions with the TerraSAR-X and TanDEM-X satellites have been made. The master image has been selected following the criterion of minimizing the perpendicular baseline, in order to minimize the (range) spectral decorrelation.

Fig. 16 shows interferometric results for the pairs acquired on $07 / 08 / 2016$ and 20/05/2017, in descending geometry, with a temporal baseline of about 9.5 months. Southern Pakistan is arid and has very little vegetation; therefore, interferograms remained coherent through the observation period. The coherence (a) and the phases of both looks (b) (c) after topographic phase removal are shown, where strong turbulent tropospheric perturbations can be appreciated. The differential phase between looks (d) is almost not affected by the turbulent troposphere. The along-track deformation allows clearly to identify two areas displacing in opposite directions, which confirms post-seismic activity.

The mean azimuth velocity can be estimated applying the periodogram operator, introduced in (34), to the multilooked ESD phases of the stack. We define an output resolution for the mean azimuth velocity of $100 \mathrm{~m} \times 100 \mathrm{~m}$, which results roughly in an equivalent number of looks of 1000 , similarly as the number of looks assumed in the computation of the performances in section IV.

The middle part of Fig. 17 shows the estimated mean azimuth velocity and temporal coherence for the ascending (a) and descending (b) geometries. Two rectangles over homogeneous
TABLE V

MEAN DOPPLER FREQUENCY DIFFERENCES AT THE OVERLAP AREA AND CORRESPONDING ESD AMBIGUITY BANDS. TOPS2 ACQUISITION WITH TWO WIDE SUBSWATHS OVER PAKISTAN.

\begin{tabular}{lcc}
\hline & SS1 & SS2 \\
\hline$\left\langle\Delta f_{\mathrm{d}}^{\text {ovl }}\right\rangle[\mathrm{Hz}]$ & 3334 & 3218 \\
Ambiguity band $[\mathrm{m}]$ & \pm 1.06 & \pm 1.10 \\
\hline
\end{tabular}

areas have been selected to compute the histograms and the mean and the standard deviation of the estimated mean deformation velocity. The bottom part of the same figure shows the resulting histograms and Gaussian fitting for each rectangle. Points with a temporal coherence greater than 0.5 have been considered. In the ascending geometry we obtain standard deviations of about 2.9 to $3.5 \mathrm{~mm} / \mathrm{month}$, whereas the expected standard deviation for 53 images is approximately $2.2 \mathrm{~mm} / \mathrm{month}$ according to the TOPS 2 curves of Fig 12. For the descending geometry the obtained standard deviation is below $2.5 \mathrm{~mm} / \mathrm{month}$, whereas $1.7 \mathrm{~mm} / \mathrm{month}$ is expected in case of having 67 images, according to the theoretical curves. The differences between expected and measured standard deviations are very likely due to a mismatch of the decorrelation model used for the calculation of the expected performance, as well as due to the inherent assumptions of the HCRB not occurring in a real scenario.

Fig. 18 shows the geocoded maps of the estimated mean azimuth velocities for the descending (a) and ascending geometries (b). The black line indicates the Surface rupture line derived from Landsat pixel offset tracking applied to co-seismic images of the $2013 \mathrm{M}_{\mathrm{w}} 7.7$ Earthquake. For both geometries the post-seismic azimuth deformation map presents two areas with clear different behavior at both sides of this line, indicating that the post-seismic deformation follows a similar pattern to the co-seismic one. The same conclusion has been reported from Sentinel-1 time-series analyses in the line-of-sight direction [45], which states that the surface displacements are dominated by horizontal motion of a similar sense to the 2013 earthquake.

The area on the north side of the rupture line is moving towards South-West, a fact which is clearly visible on both geometries, since the motion is quite well aligned with the along-track geometry. On the other hand, the projection of the motion in the southern area is greater for the ascending acquisition.

It is interesting to note that the maximum along-track deformation velocity is about $2.5 \mathrm{~mm} / \mathrm{month}$, which for the largest temporal baseline in the current data set corresponds to an ESD phase of just $20^{\circ}$. Thanks to the long-term coherence and the exploitation of the time series it is possible to perform a reliable estimation of the deformation.

\section{CONCLUSION}

Burst-mode acquisitions have been traditionally employed to map large areas by mapping different range sub- 


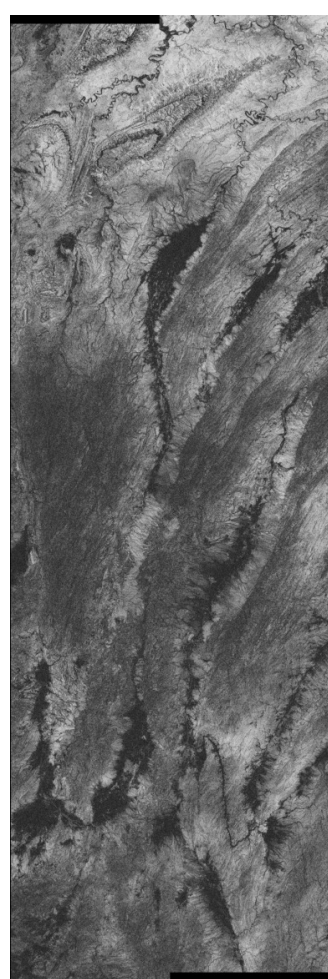

(a) Interferometric coherence

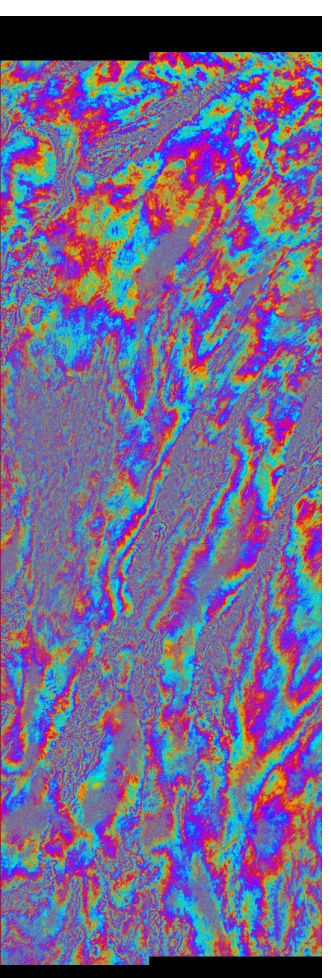

(b) InSAR phase look1

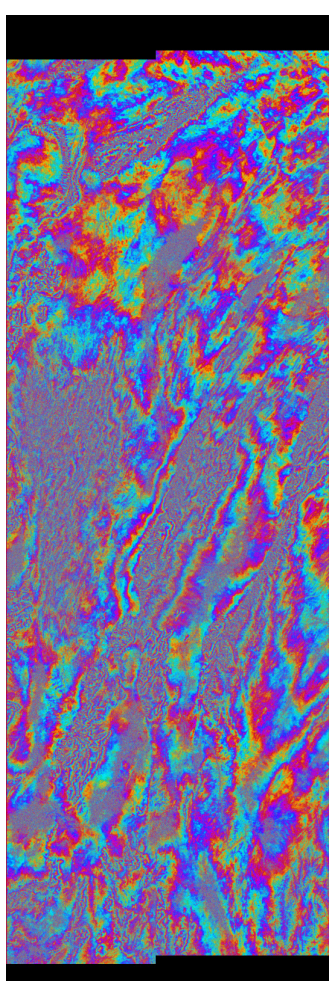

(c) InSAR phase look2

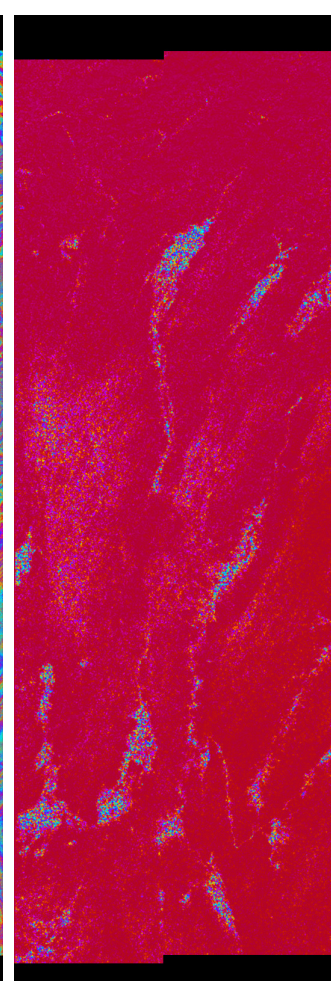

(d) ESD phase

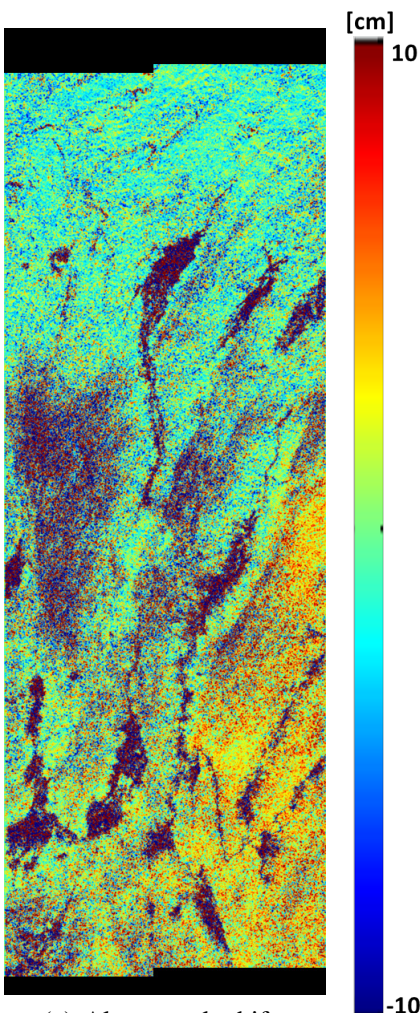

(e) Along-track shift

Fig. 16. Interferometric TOPS2 results over Balochistan site corresponding to the pair 07/08/2016 - 20/05/2017 (approx. 9.5 months), acquired in descending geometry. The acquisitions cover an area of $85 \mathrm{~km} \times 265 \mathrm{~km}$ and employ two subswaths with an azimuth resolution of $17 \mathrm{~m}$. The (a) interferometric coherence, (b) phase of look 1 and (c) look 2 after topographic phase removal, (d) differential phase and the (e) along-track shift map are shown. The phases are scaled between $\pm 180^{\circ}$. Range is horizontal and azimuth is vertical.

swaths. There have been multiple radar missions which have operated in ScanSAR mode, as e.g. RADARSAT-1, ENVISAT-ASAR, ALOS-PALSAR, or are currently operating, e.g. RADARSAT-2, ALOS-2-PALSAR, COSMO-SkyMed and TerraSAR-X. The design of the modes has been done employing multiple looks due to SNR reasons (except TerraSAR-X ScanSAR, which is a 1-look system). In this way the dependence of the azimuth target position could be moderately mitigated. The TOPS mode solved the scalloping and associated effects, making the use of multiple looks not necessary. However, if we compare modes recording bursts of echoes to full aperture modes (StripMap), the sensitivity to the along-track direction is degraded due to its lower azimuth resolution.

In this contribution we have proposed the 2-look TOPS concept, with the main motivation of overcoming this limitation. The 2-look TOPS mode achieves the same coverage as a single look TOPS mode at the expense of a degradation of the azimuth resolution by a factor of two, maintaining however the number of looks for a given output resolution product. Two different design principles have been presented, the first one, TOPS2, follows a design based on resolution and achieves very similar azimuth sensitivity to StripMap. The second one, TOPS2+, consists in an optimization of the 2-look TOPS mode, which achieves an enhanced azimuth sensitivity. The design is based on selecting larger antenna beam steering angles and is mainly limited by azimuth ambiguities. A quantitative analysis of the ambiguities impact has been provided, including a criterion to select optimal acquisition PRFs. The range ambiguities have been, as well, considered in order to choose the optimal PRF in terms of total ambiguities. For the sake of example a maximum steering angle of $1.1^{\circ}$ has been chosen taking TerraSAR-X system parameters, which delivers a gain in the estimation of the azimuth motion of $11 \mathrm{~dB}$ (relative variance) with respect to StripMap mode. The total ambiguity-to-signal ratio is approximately $-21 \mathrm{~dB}$ in average (over all subswaths). In case this ratio is not acceptable, either the maximum steering can be relaxed or the processed Doppler bandwidth reduced. We have obtained a gain of about $1 \mathrm{~dB}$ when reducing the processed bandwidth by $30 \%$.

The looks can be combined at the interferometric processing stage reducing this way the residual scalloping. In the case of TOPS2, the resulting residual scalloping is $0.07 \mathrm{~dB}$, whereas for TOPS2+ it amounts $0.4 \mathrm{~dB}$ (similar to TOPS, i.e., $0.36 \mathrm{~dB}$ ). An additional benefit is that ionospheric perturbations can be estimated with continuous coverage (in contrast to TOPS).

The 2-D expected performances in the deformation estimation employing acquisition pairs or time series have been presented, as well as 3-D performances combining ascending and descending geometries. Assuming the TerraSAR-X system and an exponential decorrelation model with a time constant of 40 days a long-term coherence of 0.1 and a power of the turbulent troposphere of $1 \mathrm{~cm}^{2}$, we would need 30 TOPS2 images to obtain an accuracy in the mean azimuth velocity of about 
(a) Ascending

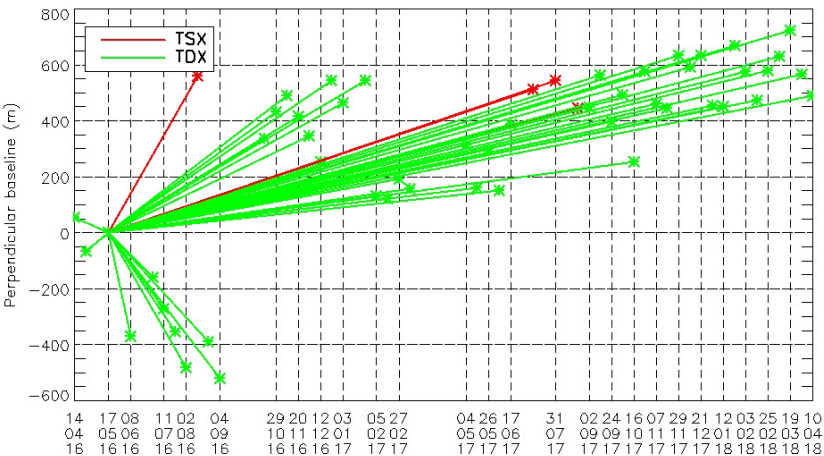

Mean azimuth velocity $[\mathrm{cm} / \mathrm{month}]$
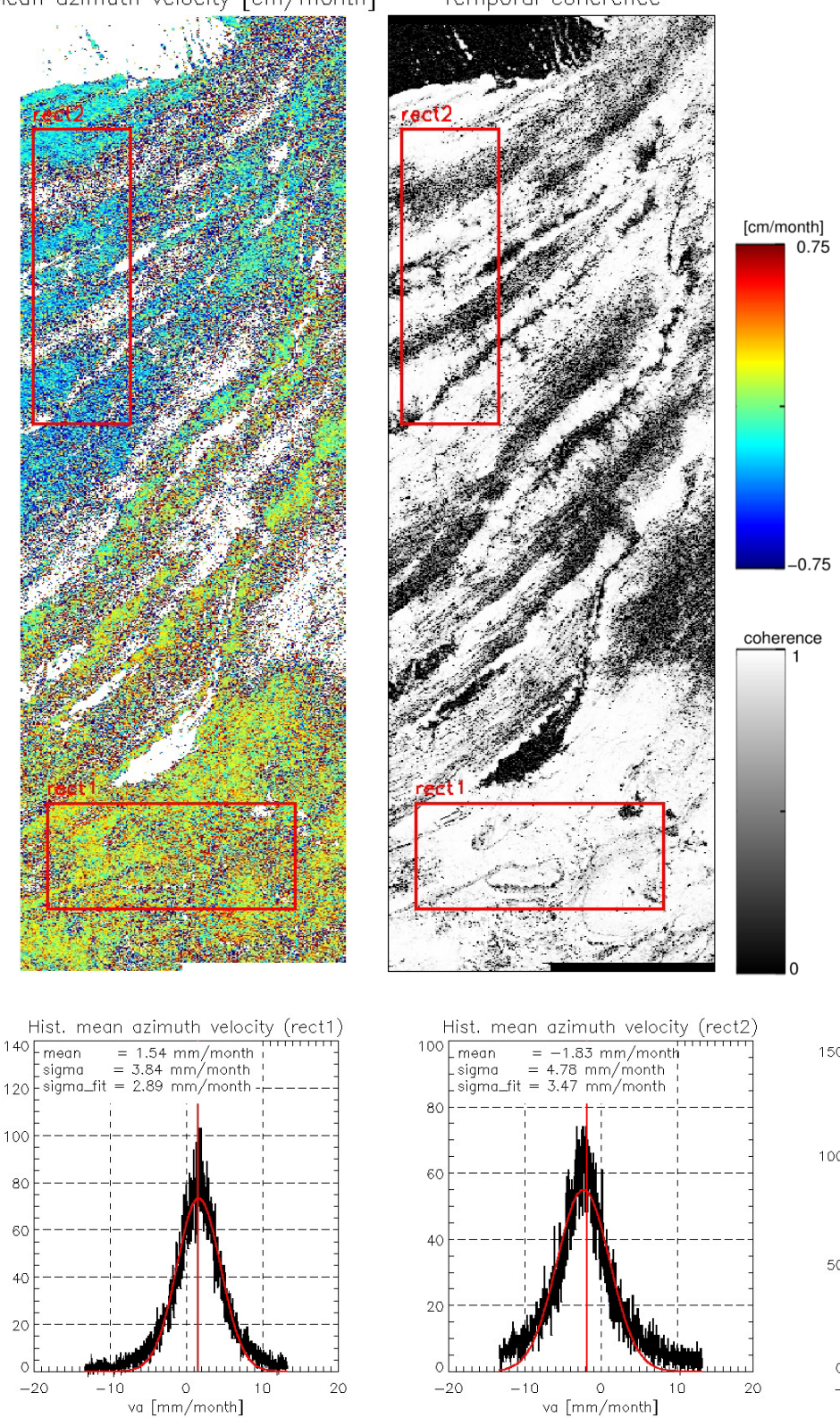

(b) Descending
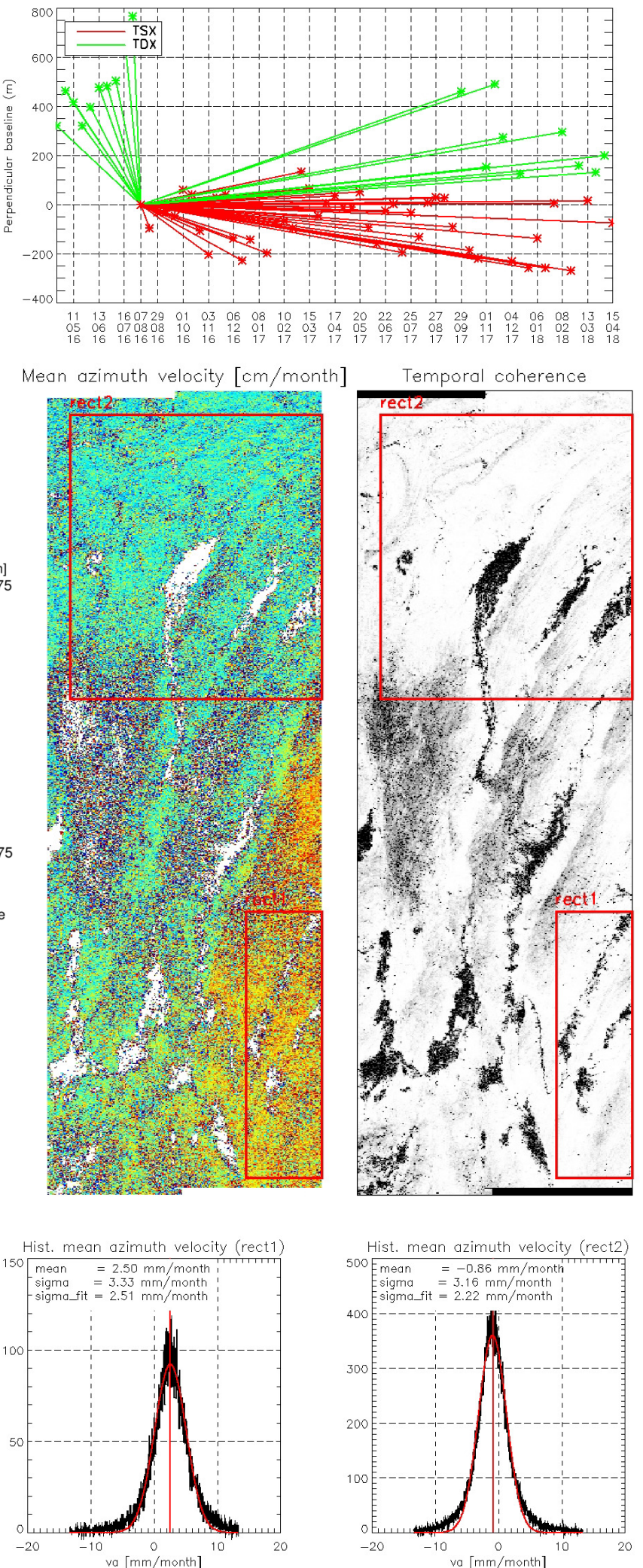

Fig. 17. Time-series TOPS2 results over Balochistan site corresponding to the time frame April 2016 to April 2018. The left part corresponds to the ascending geometry, with a total of 53 images, whereas the right part to the descending geometry, having a total of 67 images. The top part shows the distribution of the perpendicular and temporal baselines for both geometries. The middle part of the figure shows the retrieved mean azimuth velocity map and the temporal coherence, indicating the areas covered by two rectangles for statistics analysis. Range is horizontal and azimuth is vertical. The bottom part shows the histograms of the mean velocity, where a Gaussian fitting (red line) has been performed. The average mean velocity and the standard deviation are provided, as well as the standard deviation result from the Gaussian fit. 
(a) Ascending

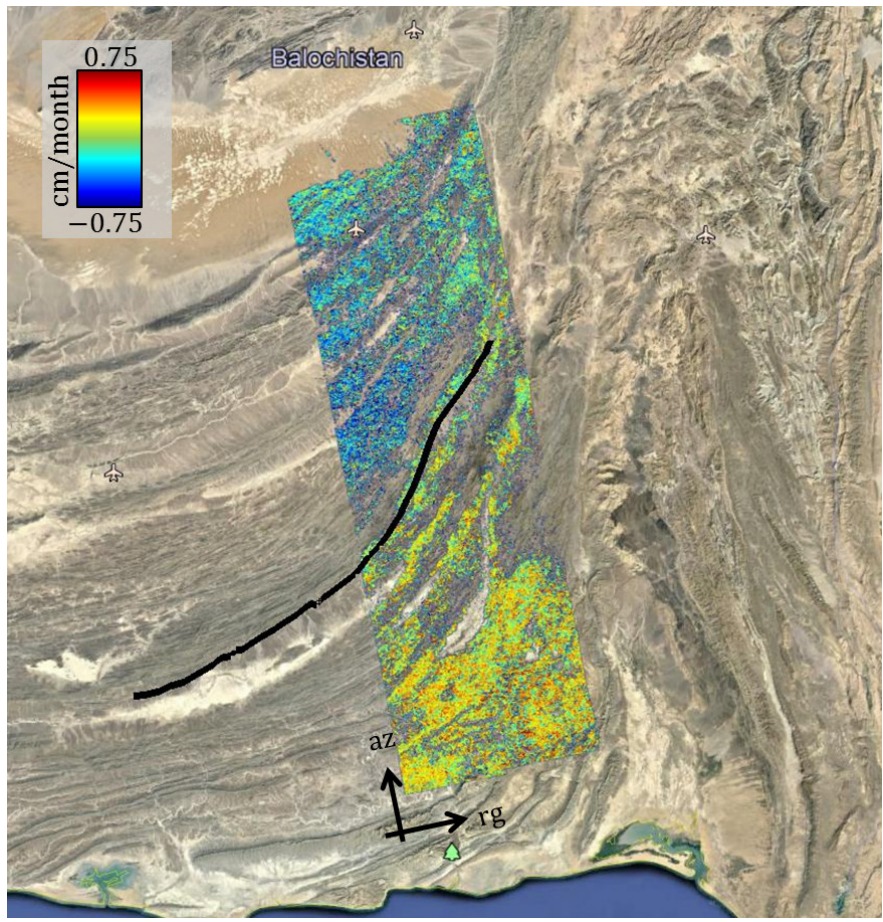

(b) Descending

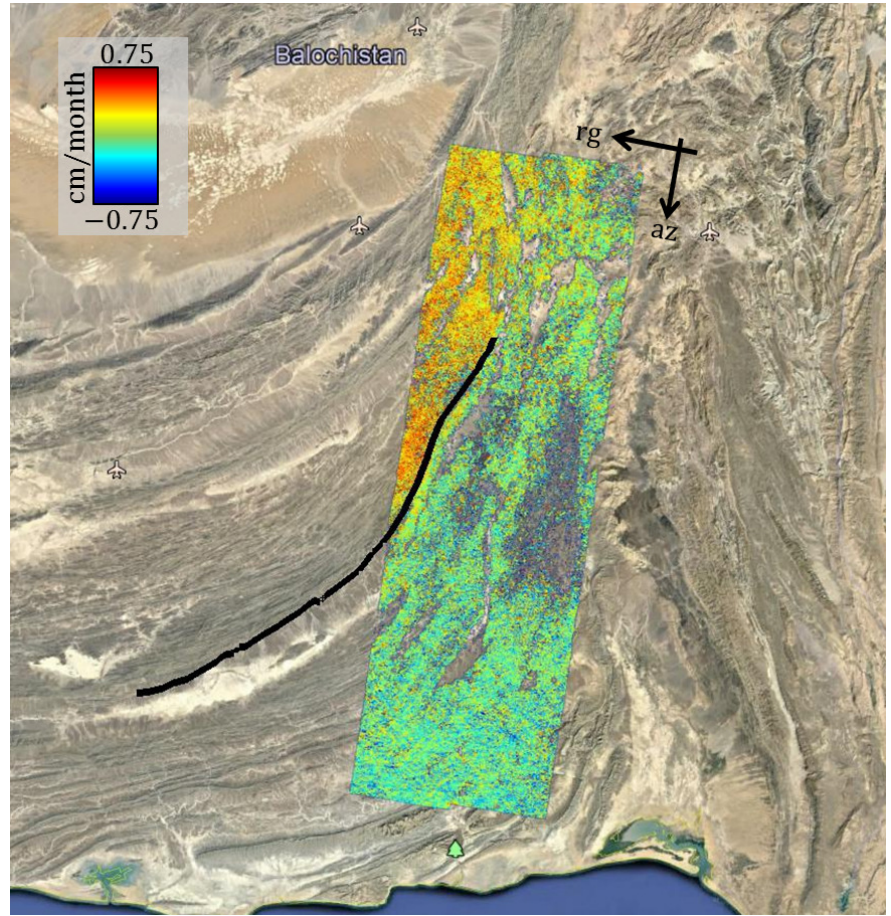

Fig. 18. Result on the estimation of the mean azimuth velocities over Balochistan employing time-series for the (a) ascending and (b) descending geometries. The black line indicates the surface rupture line derived from Landsat pixel offset tracking applied to co-seismic images of the 2013 $\mathrm{M}_{\mathrm{w}} 7.7$ earthquake. For both geometries the post-seismic azimuth deformation map presents two areas with clear different behavior at both sides of this line indicating that the post-seismic deformation follows a similar pattern to the co-seismic one.

$4 \mathrm{~mm} / \mathrm{month}$. In case of using TOPS $2+$ the number of images is reduced to 13 for the same accuracy. Same conclusions for the N-S direction can be drawn, when combining acquisitions in ascending and descending geometry.

The methodologies to derive the azimuth displacement have been described and applied to two different scenarios, using real data from TerraSAR-X, with fast deformation (glacier flow) and slow deformation (post-seismic ground displacement). In the first case we have shown the high potential of the exploitation of 2-look modes to perform a local coregistration, removing in that case phase discontinuities due to azimuth displacement. The second case demonstrates the retrieval of a post-seismic signal in along-track direction employing time-series, with a 2-year stack, delivering accuracies in the mean along-track velocity of a few $\mathrm{mm} / \mathrm{month}$.

We would like to stress that the 2-look concept employing burst modes is not only restricted to TOPS operation but can also be employed with ScanSAR. In this case a design based on the azimuth sensitivity, as done for TOPS $2+$, is not possible, since the maximum Doppler span is linked solely to the antenna beamwidth. However, in spite of achieving a lower sensitivity than its equivalent TOPS mode, spectral diversity techniques can be also applied.

This contribution has demonstrated the benefits and high potential of the 2-look TOPS concept, specially, for geophysical applications, where high azimuth resolution is not required.

\section{ACKNOWLEDGMENTS}

The authors would like to thank Carolina González, from DLR, for providing the routines for the calculation of the range ambiguities. We thank Ken Hudnut, from Caltech and Eric Fielding, Jet Propulsion Laboratory, for providing the surface rupture line of the 2013 co-seismic event and for discussions regarding the Balochistan site. We would also to thank the anonymous reviewers for their constructive comments and suggestions, which improved the readability of this paper. The employed TerraSAR-X images have been provided by DLR under proposal MTH0484.

\section{REFERENCES}

[1] T. J. Wright, B. E. Parsons, and Z. Lu, "Toward mapping surface deformation in three dimensions using InSAR,' Geophysical Research Letters, vol. 31, no. 1, pp. n/a-n/a, 2004, 101607. [Online]. Available: http://dx.doi.org/10.1029/2003GL018827

[2] R. Michel, J. P. Avouac, and J. Taboury, "Measuring ground displacements from SAR amplitude images: Application to the Landers earthquake," Geophys. Res. Lett., 26(7), 875878., 1999.

[3] T. Strozzi, A. Luckman, T. Murray, U. Wegmuller, and C. L. Werner, "Glacier motion estimation using SAR offset-tracking procedures," IEEE Transactions on Geoscience and Remote Sensing, vol. 40, no. 11, pp. 2384-2391, Nov 2002.

[4] K. Tomiyasu, "Conceptual Performance of a Satellite Borne, Wide Swath Synthetic Aperture Radar," IEEE Transactions on Geoscience and Remote Sensing, vol. GE-19, no. 2, pp. 108-116, April 1981.

[5] F. De Zan and A. Monti Guarnieri, "TOPSAR: Terrain Observation by Progressive Scans," Geoscience and Remote Sensing, IEEE Transactions on, vol. 44, no. 9, pp. 2352-2360, Sept 2006. 
[6] R. Bamler and M. Eineder, "Accuracy of differential shift estimation by correlation and split-bandwidth interferometry for wideband and delta-k SAR systems," Geoscience and Remote Sensing Letters, IEEE, vol. 2, no. 2, pp. 151-155, April 2005.

[7] M. Suess, B. Grafmueller, and R. Zahn, "A novel high resolution, wide swath SAR system," in IGARSS 2001. Scanning the Present and Resolving the Future. Proceedings. IEEE 2001 International Geoscience and Remote Sensing Symposium (Cat. No.01CH37217), vol. 3, 2001, pp. 1013-1015 vol.3.

[8] M. Younis, C. Fischer, and W. Wiesbeck, "Digital beamforming in SAR systems," IEEE Transactions on Geoscience and Remote Sensing, vol. 41, no. 7, pp. 1735-1739, July 2003.

[9] G. Krieger, N. Gebert, and A. Moreira, "Unambiguous SAR signal reconstruction from nonuniform displaced phase center sampling," IEEE Geoscience and Remote Sensing Letters, vol. 1, no. 4, pp. 260-264, Oct 2004.

[10] N. Gebert, G. Krieger, and A. Moreira, "Digital Beamforming on Receive: Techniques and Optimization Strategies for High-Resolution Wide-Swath SAR Imaging," IEEE Transactions on Aerospace and Electronic Systems, vol. 45, no. 2, pp. 564-592, April 2009.

[11] M. Villano, G. Krieger, and A. Moreira, "Staggered SAR: HighResolution Wide-Swath Imaging by Continuous PRI Variation," IEEE Transactions on Geoscience and Remote Sensing, vol. 52, no. 7, pp. 4462-4479, July 2014.

[12] R. Scheiber and A. Moreira, "Coregistration of interferometric SAR images using spectral diversity," Geoscience and Remote Sensing, IEEE Transactions on, vol. 38, no. 5, pp. 2179-2191, Sep 2000.

[13] B. D. Bechor and H. A. Zebker, "Measuring two-dimensional movements using a single InSAR pair," Geophys. Res. Lett., vol. 33, no. 16, August 2006.

[14] P. Prats-Iraola, M. Rodriguez-Cassola, N. Yague-Martinez, P. LopezDekker, R. Scheiber, F. D. Zan, T. Kraus, and S. Wollstadt, "Repeatpass interferometric experiments with the TanDEM-X constellation for accurate along-track motion estimation," in 2015 IEEE International Geoscience and Remote Sensing Symposium (IGARSS), July 2015, pp. 4077-4080.

[15] P. Prats-Iraola, N. Yague-Martinez, S. Wollstadt, T. Kraus, and R. Scheiber, "Demonstration of the Applicability of 2-Look Burst Modes in Non-Stationary Scenarios with TerraSAR-X," in Proceedings of EUSAR 2016: 11th European Conference on Synthetic Aperture Radar, June 2016, pp. 1-6.

[16] S. J. Frasier and A. J. Camps, "Dual-beam interferometry for ocean surface current vector mapping," IEEE Transactions on Geoscience and Remote Sensing, vol. 39, no. 2, pp. 401-414, Feb 2001.

[17] P. Prats-Iraola, R. Scheiber, L. Marotti, S. Wollstadt, and A. Reigber "TOPS Interferometry with TerraSAR-X," Geoscience and Remote Sensing, IEEE Transactions on, vol. 50, no. 8, pp. 3179-3188, Aug 2012.

[18] F. De Zan, P. Prats-Iraola, R. Scheiber, and A. Rucci, "Interferometry with TOPS: coregistration and azimuth shifts," in EUSAR 2014; 10th European Conference on Synthetic Aperture Radar; Proceedings of, June 2014, pp. 1-4.

[19] R. Bamler and M. Eineder, "ScanSAR processing using standard high precision SAR algorithms," IEEE Transactions on Geoscience and Remote Sensing, vol. 34, no. 1, pp. 212-218, Jan 1996.

[20] U. Steinbrecher, T. Kraus, G. C. Alfonzo, C. Grigorov, D. Schulze, and B. Braeutigam, "TerraSAR-X: Design of the new operational WideScanSAR mode," in EUSAR 2014; 10th European Conference on Synthetic Aperture Radar, June 2014, pp. 1-4.

[21] A. Meta, J. Mittermayer, P. Prats, R. Scheiber, and U. Steinbrecher, "TOPS Imaging With TerraSAR-X: Mode Design and Performance Analysis," Geoscience and Remote Sensing, IEEE Transactions on, vol. 48, no. 2, pp. 759-769, Feb 2010.

[22] J. Mittermayer, S. Wollstadt, P. Prats-Iraola, P. Lopez-Dekker G. Krieger, and A. Moreira, "Bidirectional SAR Imaging Mode," IEEE Transactions on Geoscience and Remote Sensing, vol. 51, no. 1, pp. 601-614, Jan 2013.
[23] J. Curlander and R. Mcdonough, Synthetic Aperture Radar: Systems and Signal Processing. Wiley, New York, 1991.

[24] F. Ulaby and C. Dobson, Handbook of Radar Scattering: Statistics for Terrain. Norwood, MA, USA: Artech House, 1989.

[25] J. O. Hagberg, L. M. H. Ulander, and J. Askne, "Repeat-Pass SAR Interferometry Over Forested Terrain," IEEE Transactions on Geoscience and Remote Sensing, vol. 54, no. 4, pp. 2220-2234, April 1995.

[26] H. A. Zebker and J. Villasenor, "Decorrelation in interferometric radar echoes," IEEE Transactions on Geoscience and Remote Sensing, vol. 30, no. 5, pp. 950-959, Sep 1992.

[27] R. F. Hanssen, Radar Interferometry: Data interpretation and error analysis. Delft University of Technology. The Netherlands, 2001.

[28] P. Prats-Iraola, P. Lopez-Dekker, F. D. Zan, N. Yagüe-Martínez, M. Zonno, and M. Rodriguez-Cassola, "Performance of 3-D Surface Deformation Estimation for Simultaneous Squinted SAR Acquisitions," IEEE Transactions on Geoscience and Remote Sensing, vol. PP, no. 99, pp. $1-12,2017$.

[29] A. M. Guarnieri and S. Tebaldini, "Hybrid Cramér-Rao Bounds for Crustal Displacement Field Estimators in SAR Interferometry," IEEE Signal Processing Letters, vol. 14, no. 12, pp. 1012-1015, Dec 2007.

[30] F. Rocca, "Modeling Interferogram Stacks," Geoscience and Remote Sensing, IEEE Transactions on, vol. 45, no. 10, pp. 3289-3299, Oct 2007.

[31] G. Gomba, F. Rodríguez-González, and F. D. Zan, "Ionospheric Phase Screen Compensation for the Sentinel-1 TOPS and ALOS-2 ScanSAR Modes," IEEE Transactions on Geoscience and Remote Sensing, vol. 55, no. 1, pp. 223-235, Jan 2017.

[32] F. Meyer, R. Bamler, N. Jakowski, and T. Fritz, "The Potential of LowFrequency SAR Systems for Mapping Ionospheric TEC Distributions," IEEE Geoscience and Remote Sensing Letters, vol. 3, no. 4, pp. 560564, Oct 2006

[33] G. Gomba and F. D. Zan, "Bayesian Data Combination for the Estimation of Ionospheric Effects in SAR Interferograms," IEEE Transactions on Geoscience and Remote Sensing, vol. 55, no. 11, pp. 6582-6593, Nov 2017.

[34] N. Yague-Martinez, P. Prats-Iraola, F. R. Gonzalez, R. Brcic, R. Shau, D. Geudtner, M. Eineder, and R. Bamler, "Interferometric Processing of Sentinel-1 TOPS Data," IEEE Transactions on Geoscience and Remote Sensing, vol. 54, no. 4, pp. 2220-2234, April 2016.

[35] S. Mancon, A. M. Guarnieri, D. Giudici, and S. Tebaldini, "On the Phase Calibration by Multisquint Analysis in TOPSAR and Stripmap Interferometry," IEEE Transactions on Geoscience and Remote Sensing, vol. 55, no. 1, pp. 134-147, Jan 2017.

[36] E. Sansosti, P. Berardino, M. Manunta, F. Serafino, and G. Fornaro, "Geometrical SAR image registration," Geoscience and Remote Sensing, IEEE Transactions on, vol. 44, no. 10, pp. 2861-2870, Oct 2006.

[37] F. D. Zan, P. Prats-Iraola, and M. Rodriguez-Cassola, "On the Dependence of Delta-k Efficiency on Multilooking," IEEE Geoscience and Remote Sensing Letters, vol. 12, no. 8, pp. 1745-1749, Aug 2015.

[38] R. Bamler and D. Just, "Phase statistics of interferograms with application to Synthetic Aperture Radar," Applied Optics, vol. 33, issue 20, pp. 4361-4368, July 1994.

[39] A. Monti Guarnieri and S. Tebaldini, "On the Exploitation of Target Statistics for SAR Interferometry Applications," Geoscience and Remote Sensing, IEEE Transactions on, vol. 46, no. 11, pp. 3436-3443, Nov 2008.

[40] O. Mora, J. J. Mallorqui, and A. Broquetas, "Linear and nonlinear terrain deformation maps from a reduced set of interferometric SAR images," IEEE Transactions on Geoscience and Remote Sensing, vol. 41, no. 10, pp. 2243-2253, Oct 2003.

[41] T. Nagler, H. Rott, M. Hetzenecker, J. Wuite, and P. Potin, "The Sentinel-1 Mission: New Opportunities for Ice Sheet Observations," Remote Sensing, vol. 7, no. 7, pp. 9371-9389, 2015. [Online]. Available: http://www.mdpi.com/2072-4292/7/7/9371 
[42] I. M. Howat, A. Negrete, and B. E. Smith, "The Greenland Ice Mapping Project (GIMP) land classification and surface elevation data sets," The Cryosphere, vol. 8, no. 4, pp. 1509-1518, 2014. [Online]. Available: https://www.the-cryosphere.net/8/1509/2014/

[43] J.-P. Avouac, F. Ayoub, S. Wei, J.-P. Ampuero, L. Meng, S. Leprince, R. Jolivet, Z. Duputel, and D. Helmberger, "The 2013, Mw 7.7 Balochistan earthquake, energetic strike-slip reactivation of a thrust fault," Earth and Planetary Science Letters, 2014.

[44] R. Jolivet, Z. Duputel, B. Riel, M. Simons, L. Rivera, S. E. Minson, H. Zhang, M. A. G. Aivazis, F. Ayoub, S. Leprince, S. Samsonov, M. Motagh, and E. J. Fielding, "The 2013 Mw 7.7 Balochistan earthquake : Seismic potential of an accretionary wedge," Bulletin of the Seismological Society of America, Seismological Society of America., 2014.

[45] K. E. Peterson, W. D. Barnhart, and S. Li, "Viscous Accretionary Prisms: Viscoelastic Relaxation of the Makran Accretionary Prism Following the 2013 Baluchistan, Pakistan Earthquake," Journal of Geophysical Research: Solid Earth, 17 October 2018. [Online]. Available: https://doi.org/10.1029/2018JB016057

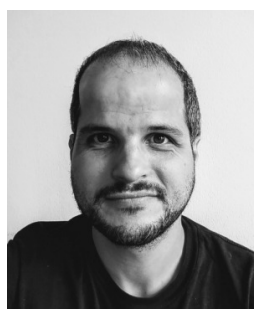

Néstor Yagüe-Martínez received the Ingeniero degree in Telecommunication Engineering from the Universitat Politècnica de València (UPV), Valencia, Spain, in 2004. From 2006 to 2015 he was with the Chair for Remote Sensing Technology, Technische Universität München (TUM), Munich, Germany, and also with the Remote Sensing Technology Institute, German Aerospace Center (DLR), Oberpfaffenhofen, Germany, where he worked in the development of the operational interferometric processor of the TanDEM-X mission. He spent a year from 2011 to 2012 at Starlab Barcelona S.L. working on alongtrack Interferometry for ocean surface currents retrieval. In 2015 he joined the Multimodal Algorithms Group, Microwaves and Radar Institute, DLR, Oberpfaffenhofen. His research interests include SAR and InSAR processing and the development of advanced interferometric acquisition modes for the measurement of tectonic and volcanic deformation.

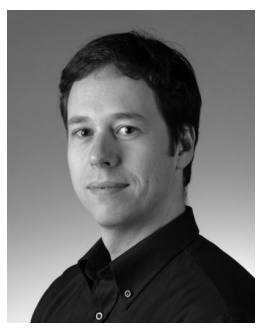

Pau Prats-Iraola (S'03-M'06-SM'13) was born in Madrid, Spain, in 1977. He received the Ingeniero degree and the $\mathrm{Ph} . \mathrm{D}$. degree, both in telecommunications engineering, from the Universitat Politècnica de Catalunya (UPC), Barcelona, Spain, in 2001 and 2006, respectively. In 2001, he was a Research Assistant at the Institute of Geomatics, Spain. In 2002, he was at the Department of Signal Theory and Communications, UPC, where he worked in the field of airborne repeat-pass interferometry and airborne differential SAR interferometry. From December 2002 to August 2006, he was an Assistant Professor at the Department of Telecommunications and Systems Engineering, Universitat Autònoma de Barcelona, Barcelona, Spain. In 2006, he joined the Microwaves and Radar Institute, German Aerospace Center (DLR), Wessling, Germany, where, since 2009, he has been the Head of the Multimodal Algorithms Group. His research interests include high-resolution airborne/spaceborne monostatic/bistatic SAR processing, SAR interferometry, advanced interferometric acquisition modes, Persistent Scatterer Interferometry (PSI), SAR tomography and end-to-end SAR simulation. He has co-authored more than 50 peer-reviewed journal papers in the field.

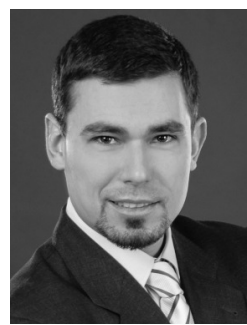

Steffen Wollstadt received the Dipl.Ing. (M.Sc.) degree in electrical engineering from the Technical University of Darmstadt, Darmstadt, Germany, in 2005 , with a thesis on metamaterial-based microstrip antenna design. In 2006, he joined the Microwaves and Radar Institute, German Aerospace Center (DLR), Oberpfaffenhofen, Germany. Until 2008, he was with the Satellite Synthetic Aperture Radar (SAR) Systems Department, as a Project Engineer in the System Engineering and Calibration part of the TerraSAR-X ground segment, where he was involved in the TerraSAR-X and TanDEM-X instrument command generation. Since 2009, he has been with the SAR Missions Research Group, Radar Concepts Department, where he has been involved in TOPS Sentinel-1 image quality and SAR performance investigations and in various experiments with TerraSAR-X and TanDEM-X about novel imaging modes and SAR performance improvement. He is responsible for the commanding of experimenta TOPS imaging mode acquisitions with TerraSAR-X. His research interests include spaceborne SAR mission and instrument analysis and interferometric problems, including along-track, repeat-pass, and differential interferometry.

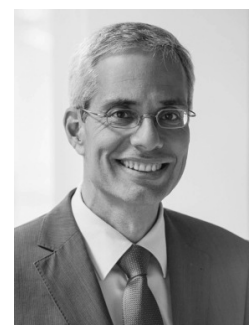

Alberto Moreira (M'92-S'96-F'04) received the B.S.E.E. and M.S.E.E. degrees from the Aeronautical Technological Institute (ITA), São José dos Campos, in 1984 and 1986, respectively, and the Eng. Dr. degree (with honors) from the Technical University of Munich, Germany, in 1993. From 1996 to 2001, he was the Chief Scientist and Engineer with the SAR Technology Department, German Aerospace Center (DLR), Oberpfaffenhofen, Germany. Under his leadership, the DLR airborne SAR system has been upgraded to operate in innovative imaging modes like polarimetric SAR interferometry and SAR tomography. Since 2001, Prof. Moreira is the Director of the Microwaves and Radar Institute at DLR and a Full Professor with the Karlsruhe Institute of Technology (KIT), in the field of microwave remote sensing. His DLR's Institute contributes to several scientific programs and projects for spaceborne SAR missions like TerraSAR-X, TanDEM-X, SARLupe and SARah as well as Kompsat-6, PAZ, Sentinel-1, BIOMASS and Tandem-L. The mission TanDEM-X, led by his Institute, has generated a global, high-resolution digital elevation model of the Earth with unprecedented accuracy. Prof. Moreira is the initiator and principal investigator (PI) for this mission. His professional interests and research areas encompass spaceborne radar end-to-end system design, microwave techniques and system concepts, signal processing, and remote sensing applications. Prof. Moreira is author or co-author of more than 400 publications in international conferences and journals, 8 book chapters, and holds 41 international patent grants in the radar and antenna field. He is an IEEE fellow and has served as President of the IEEE Geoscience and Remote Sensing (GRS) Society in 2010. He was founder and chair of the GRSS German Chapter (20032008), served as Associate Editor for the IEEE GRS Letters (2003-2007) and for the IEEE TGRS (since 2005), and is serving as chair of the Major Awards of the GRS society since 2017. He is recipient of several international awards including the IEEE AESS Nathanson Award (1999) for the Young Radar Engineer of the Year, the IEEE Kiyo Tomiyasu Field Award (2007), IEEE W.R.G. Baker Award from the IEEE Board of Directors (2012), and the IEEE GRSS Distinguished Achievement Award (2014). He and his colleagues received the GRSS Transactions Prize Paper Awards in 1997, 2001, and 2007 and the GRSS Letters Prize Paper Award in 2015 and 2017. From 2012 to 2017 he has served as principal investigator for the Helmholtz Alliance "Remote Sensing and Earth System Dynamics" and is currently the PI for Tandem-L, a radar mission proposal for the global observation of dynamic processes on Earth's surface with unprecedented quality and resolution. 